\title{
RATAN-600 7.6-cm Deep Sky Strip Surveys at the Declination of the SS433 Source During the 1980-1999 Period. Data Reduction and the Catalog of Radio Sources in the Right-Ascension Interval $7^{h} \leq$ R.A. $<17^{h}$
}

\author{
N. S. Soboleva, ${ }^{1}$ E. K. Majorova,${ }^{2}$ O. P. Zhelenkova, ${ }^{2}$ A. V. Temirova, ${ }^{1}$ and N. N. Bursov ${ }^{2}$ \\ ${ }^{1}$ St. Petersburg Branch of the Special Astrophysical Observatory, \\ Russian Academy of Sciences, Pulkovo, St. Petersburg, 196140 Russia \\ ${ }^{2}$ Special Astrophysical Observatory of the Russian AS, Nizhnij Arkhyz 369167, Russia
}

\begin{abstract}
We use two independent methods to reduce the data of the surveys made with RATAN600 radio telescope at $7.6 \mathrm{~cm}$ in $1988-1999$ at the declination of the SS433 source. We also reprocess the data of the "Cold" survey (1980-1981). The resulting RCR (RATAN COLD REFINED) catalog contains the right ascensions and fluxes of objects identified with those of the NVSS catalog in the right-ascension interval $7^{h} \leq$ R.A. $<17^{h}$. We obtain the spectra of the radio sources and determine their spectral indices at 3.94 and $0.5 \mathrm{GHz}$. The spectra are based on the data from all known catalogs available from the CATS, Vizier, and NED databases, and the flux estimates inferred from the maps of the VLSS and GB6 surveys. For 245 of the 550 objects of the RCR catalog the fluxes are known at two frequencies only: $3.94 \mathrm{GHz}(\mathrm{RCR})$ and $1.4 \mathrm{GHz}$ (NVSS). These are mostly sources with fluxes smaller than $30 \mathrm{mJy}$. About $65 \%$ of these sources have flat or inverse spectra $(\alpha>-0.5)$. We analyze the reliability of the results obtained for the entire list of objects and construct the histograms of the spectral indices and fluxes of the sources. Our main conclusion is that all $10-15 \mathrm{mJy}$ objects found in the considered right-ascension interval were already included in the decimeter-wave catalogs.
\end{abstract}

DOI:10.1134/S1990341310010050

\section{INTRODUCTION}

In 1991 by Parijskij et al. [1] a catalog of 7.6cm radio sources in the $4^{h} \leq$ R.A. $<22^{h}$ interval of right ascensions (RC-catalog) and 31-cm radio sources in the $4^{h} \leq$ R.A. $<13^{h}$ interval at the declination of $D e c_{2000}=5^{\circ} \pm 20^{\prime}$ was published. This catalog was based on the results of observations made in 1980-1981 with the RATAN-600 radio telescope in the meridian and azimuth of $30^{\circ}[2-4]$. The coordinate calibration was based on the then most accurate UTRAO $(365 \mathrm{MHz})$ catalog ${ }^{1}$.

After the publication of the NVSS and FIRST catalogs (1.4 GHz, VLA) $[5,6]$ the objects of the $\mathrm{RC}$ catalog were compared with the objects of the former two catalogs and $20-25 \%$ of $\mathrm{RC}$ objects proved to be impossible to cross identify

\footnotetext{
${ }^{1}$ The data for the sky strip of interest was kindly pro-
} vided to us by Prof. J. N. Douglas prior to publication. 
with NVSS objects [7].

Several additional observation sets were carried out from 1987 through 1999 at the Northern sector of RATAN-600 in order to refine the RC catalog and, in particular, the fluxes and coordinates of its sources. The observations, like earlier, were made at the declination of SS433. The declination varied from cycle to cycle because of precession. However, these variations proved to be too small to allow the declinations of RCR objects to be found with sufficient accuracy. The results of the reduction of these observations in the right-ascension interval $2^{h} \leq$ R.A. $<7^{h}$ and $17^{h} \leq$ R.A. $<22^{h}$ can be found in our earlier paper [8].

In this paper we report the results of the reduction of the 7.6-cm observations made in 19871999 in the right-ascension band $7^{h} \leq$ R.A. $<$ $17^{h}$. In addition, we also report the results of our rereduction of the records obtained in the "Cold" experiment in 1980.

We uses NVSS objects to calibrate the right ascensions. We also used the declinations of sources from NVSS catalog.

Almost the entire observed region has been studied with a VLA with a resolution of $5.4^{\prime \prime}$ (the FIRST catalog, $8^{h} 11^{m} \leq$ R.A. $\left.<16^{h} 26^{m}\right)$, and the results of these observations allowed us to refine the structure of the radio sources.

We report the list of objects (the RCR cata$\log$ ) found within the right-ascension band mentioned above and identified with NVSS objects [5]. We separately discuss the reliability of the identification of our objects with the objects of the NVSS catalog and the statistical conclusions based on the rereduced RC catalog. We pay special attention to objects with peculiar spectra and to objects with fluxes known only at two frequencies: $1.4 \mathrm{GHz}$ (NVSS) and $3.94 \mathrm{GHz}$ (RCR).

\section{REDUCTION OF OBSERVATIONAL DATA}

The shape of the RATAN-600 beam pattern differs substantially from that of a paraboloid antenna [9-13]. The width of the horizontal section of the beam extends with the distance in declination from the beams center. Therefore we used two different methods for data reduction, which differed mostly by the substraction of the background on averaged scans.

We computed the background with a 80 and 20-s "smoothing window" in the first and second methods, respectively. We chose the "smoothing window" based on the computed $H P B W(\triangle D e c)$ dependences, which were tested experimentally $[13,14] \quad(H P B W$ is the beam halfwidth in the horizontal section and $\Delta D e c$ is declination difference between the section considered and the central horizontal section).

It is safe to conclude that the results obtained with the background computed and then substracted with a 80-s and 20-s "window" should filter out the radio sources that cross the beam pattern at declinations outside the $D e c_{0} \pm 50^{\prime}$ and $D e c_{0} \pm 12^{\prime}$ bands, where $D e c_{0}$ is the declination 
of the central section of the observation set.

In practice, after the substraction of the background computed with a 20-s "window" the sources at $\Delta D e c> \pm 8^{\prime}$ are not excluded completely, but only decreased their antenna temperature. No reduction of the antenna temperatures is observed for sources in the $\Delta D e c \leq \pm 8^{\prime}$ band.

The effect of the atmosphere may show up if the background is computed with a 80s "window", whereas such effects are minimal if the background is computed with a 20-s wide "window", because in this case the lowfrequency noise - and atmospheric fluctuations in particular-is filtered out.

Thus the two methods result in different systematic errors in the inferred fluxes of the radio sources. Whereas the first method may overestimate the fluxes caused by the influence of the atmosphere, the second method tends to underestimate the fluxes of distant objects.

After substraction the background level we extracted the sources from averaged scans by applying Gaussian analysis. We performed R.A. calibration using strong sources and data of the NVSS catalog. For each identified source we computed its R.A. coordinates, antenna temperature $\left(\mathrm{T}_{\mathrm{a}}\right)$, and the halfwidth of the Gaussian $\left(H P B W_{i}\right)$. In addition in the first method we drew the "zero" line within the given object before fitting the Gaussian.

\subsection{First Method}

One-dimensional averaged scans are superpositions of all sources that have crossed different horizontal sections of the beam pattern, and therefore to discriminate and identify these sources more reliably we simulated the survey using NVSS images $[5,15]$ in the same way as Majorova [16] did it for the RZF survey [17].

We then compared the normalized simulated scans with real $7.6 \mathrm{~cm}$-records after the substraction of the 80-s background. Figure 1 demonstrates examples of normalized simulated and real averaged scans. The scans in Figs. 1a and $1 \mathrm{~b}$ are normalized to the level of the signal from the sources $082056+045417$ and $114520+045526$, respectively. The y-axis is in relative units and the $\mathrm{x}$-axis gives the right ascension. Simulated scans proved to be most efficient in complex cases involving the superposition of two or more sources.

When reducing the data for each observation set we used the $\Delta D e c$ values of the NVSS sources located within the $D e c_{0} \pm 1^{\circ}$ band and the $H P B W(\triangle D e c)$ dependences. We also computed the pattern factor $k_{D N}(\Delta D e c)$ and the product $\mathrm{F}_{1.4} \times k_{D N}(\Delta D e c)$, where $\mathrm{F}_{1.4}$ is the source flux at $1.4 \mathrm{GHz}$. We computed $H P B W(\Delta D e c)$ and $k_{D N}(\Delta D e c)$ using the algorithms published by Majorova [12]. The pattern factor meant for the reduction of the response from the source as a function of its distance from the central section of the survey (or the central section of the beam pattern). This factor is used 

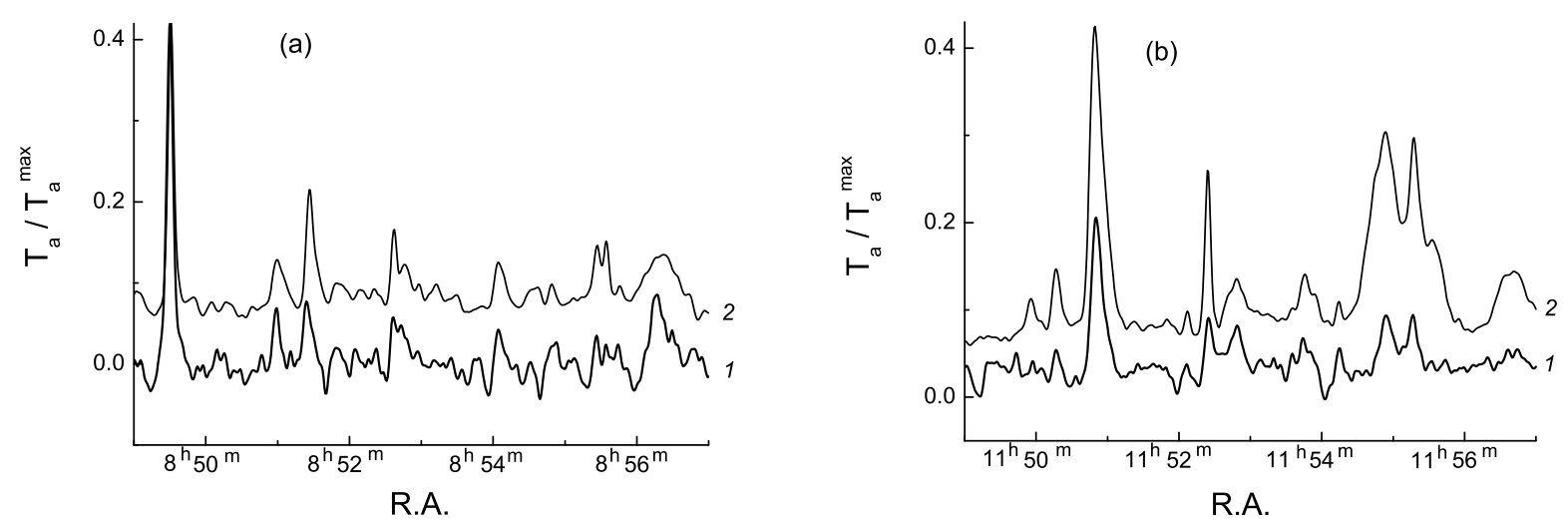

Figure 1. Normalized real averaged records of the RCR survey at $7.6 \mathrm{~cm}$ (the curves 1) and normalized simulated scans of the same sky areas based on NVSS images (the curves 2). The scans in Figs. 1a and 1b are normalized to the level of the signal from the sources $082056+045417$ and $114520+045526$, respectively.

The $\mathrm{y}$-axis is in relative units and the $\mathrm{x}$-axis gives the right ascension.

to find the fluxes of the sources. The product $\mathrm{F}_{1.4} \times k_{D N}(\Delta D e c)$ provides information about the probability of the source appearance on the real scan. This product also helps in extraction and identification of the sources with close right ascensions.

For further analysis we selected from the list of NVSS sources located within the $D e c_{0} \pm 1^{\circ}$ band those that satisfied the condition $\mathrm{F}_{1.4} \times k_{D N}(\Delta D e c)>3 . \quad$ Our $\quad$ subsequent investigation showed that no sources with $\mathrm{F}_{1.4} \times k_{D N}(\Delta D e c)<3$ had been found in the records.

A comparison of the $H P B W$ values inferred via Gaussian analysis with the computed values allowed us to control the reliability of the extraction of the sources and, in a number of cases, helped us to understand whether we found the right source in the record.

Thus, in the first method of data reduction we use three criteria to control the extraction of the sources: closeness of the R.A. of the source in the record to the R.A. of the source according to the NVSS catalog, closeness of the halfwidth of the Gaussian to its computed value, and sufficiently large value of the product $\mathrm{F}_{1.4} \times k_{D N}(\Delta D e c)$. The sensitivity varied from cycle to cycle and therefore the minimum (threshold) product also differed from cycle to cycle.

In the first method we computed the source fluxes $F_{1}$ by the following formula:

$$
\begin{aligned}
& \mathrm{F}_{1}=A \times \mathrm{T}_{\mathrm{a}} / k_{D N}, \\
& A=2 k / S_{\text {eff }}, \text { where } k \text { is the Boltzmann con- }
\end{aligned}
$$

stant and $S_{\text {eff }}$ is the effective area of the radio telescope.

We found the coefficient $A$ individually for each observation set using the technique described by Majorova and Bursov [14]. To this end, we selected sufficiently strong sources with steep spectra and known spectral indices, com- 
puted their fluxes at $7.6 \mathrm{~cm}$, and determined from the real scans the antenna temperatures of theses sources for each observation set. We then constructed the dependences $\mathrm{F} / \mathrm{T}_{\mathrm{a}}(\Delta D e c)$, that we fitted by the appropriate approximating curves using the least squares method. The coefficient $A$ is equal to the value of the approximating curve at $\Delta D e c=0$ for the observation set considered.

We computed the pattern factor $k_{D N}$ in accordance with the $\Delta D e c$ of the source. In our computations we took into account the offset of the primary horn along the focal line of the secondary mirror in the observation set considered. We then averaged the inferred fluxes over all observation sets. We list the fluxes $\mathrm{F}_{1}$ and right ascensions R.A.1 with their errors in the Table.

Note that part of the sources could be found only in one of the observation sets, in particular, in the 1980 set, that was characterized by the highest sensitivity. In some of the cases sources could be identified only in the records made in 1994, when the antenna was mounted $4^{\prime}$ lower $\left(\mathrm{H}=51^{\circ} 22^{\prime}\right)$ or higher $\left(\mathrm{H}=51^{\circ} 09^{\prime}\right)$ than the declination of SS433. The errors of the fluxes determined for sources identified in only one observation set amounts to $(20 \pm 5) \%$.

The yearly-averaged noise root-mean-squared errors in $\overline{3 \sigma}$ records are (in $\mathrm{mK}$ ): $2.2 \pm 0.5 \mathrm{mK}$ for the 1980 set; $3.4 \pm 0.4 \mathrm{mK}$ for the 1988 set; $4.9 \pm 1.7 \mathrm{mK}$ for the $1993 \mathrm{set} ; 3.2 \pm 0.4 \mathrm{mK}$ for the $1994 \mathrm{set} ; 4.5 \pm 1.7 \mathrm{mK}$ for the 1994 set $\left(\mathrm{H}=51^{\circ} 09^{\prime}\right)$, and $3.7 \pm 0.7 \mathrm{mK}$ for the 1994 set $\left(\mathrm{H}=51^{\circ} 22^{\prime}\right)$.
Figure 2 demonstrates the variations of the root-mean-squared noise error $(3 \sigma)$ (in $\mathrm{mJy}$ ) for averaged scans at different observation hours. Here $94 \mathrm{~m}$ and $94 \mathrm{p}$ correspond to the records obtained in 1994 with the antenna mounted $4^{\prime}$ below and above the declination of SS433, respectively.

The mean $\overline{3 \sigma}$ values (in mJy) averaged over all observations are equal to: $8.0 \pm 0.5 \mathrm{mJy}$ for the 1980 set; $10.6 \pm 1.3 \mathrm{mJy}$ for the 1988 set; $10.4 \pm 3.7 \mathrm{mJy}$ for the $1993 \mathrm{set} ; 9.6 \pm 1.2 \mathrm{mJy}$ for the 1994 set; $13.5 \pm 5.5 \mathrm{mJy}$ for the 1994 $\left(\mathrm{H}=51^{\circ} 09^{\prime}\right)$ set, and $11.1 \pm 2.0 \mathrm{mJy}$ for the 1994 $\left(\mathrm{H}=51^{\circ} 22^{\prime}\right)$ set. The root-mean-squared errors were computed for the scans with the 80 -s background subtracted.

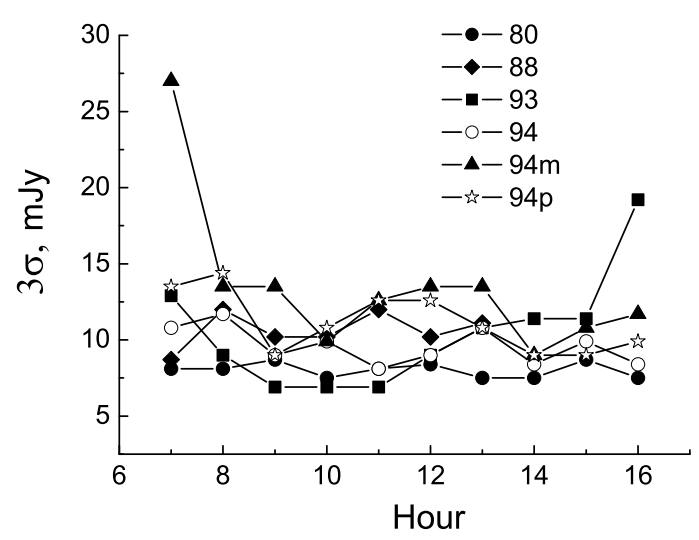

Figure 2. Root-mean-squared noise error $(3 \sigma)$ of the averaged scans as a function of the hour of observation computed for observation sets of different years. Here 94m and 94p correspond to the scans obtained in 1994 with the antenna mounted $4^{\prime}$ below and above the declination of SS433, respectively. The scans with the 80 -s background subtracted are used. 


\subsection{Second Method}

After substraction the 20-s background using the second method we applied Gaussian analysis to find the positions of the radio sources on the scan (R.A.), their antenna temperatures, and halfwidths. We then used the antenna temperatures to compute the fluxes $\mathrm{F}_{2}$ of the radio sources by the following formula:

$$
\mathrm{F}_{2}=k_{e f f} \times k_{i} \times \mathrm{T}_{\mathrm{a}} / k_{D N},
$$

where $k_{\text {eff }}$ is the factor that takes into account the effective area of the antenna and $k_{i}$ is the correcting factor to correct for the differences between the calibrations and small differences between the effective antenna areas during different observation sets. We determined $k_{i}$ from the sources with well-known spectra found within the survey band. The $k_{i}$ factors lied in the 1.1-1.5 interval depending on the year of observation. The $k_{\text {eff }}$ factor was equal to 3.5. We computed the pattern factor for each radio source with the transversal offset of the primary feed along the focal line of the secondary mirror and the vertical distance of the radio object from the central section of the beam pattern taken into account in accordance with the algorithms described by Majorova [12].

Our significance criterion is based on the presence of the object on the scans of more than two observation sets. Prior to identification of objects we smoothed the scans by applying the computed beam pattern in its central section. A detailed description of the technique can be found in [18].

After finding the fluxes and right ascensions in each observation set we computed the flux $\mathrm{F}_{2}$ and R.A.2 averaged over all observation runs and the corresponding errors (see Table).

In this method of data reduction the noise root-mean-squared error in records is somewhat lower than in the first method. This is due, first, to smoothing of records by the beam and, second, to "trimming" of noise after the subtraction of the 20-s background.

\section{CATALOG OF RADIO SOURCES AT $7.6 \mathrm{CM}$}

The inferred $7.6 \mathrm{~cm}$ fluxes and right ascensions of the sources are given in the catalog whose fragment we list in the Table.

Column 1 gives the J2000.0 coordinates of the sources according to the NVSS catalog; columns 2 and 3, the right-ascension differences of the objects between the NVSS coordinates and our estimates $\Delta \mathrm{RA}_{1}$ (the first method proposed by Majorova) and $\Delta \mathrm{RA}_{2}$ (the second method proposed by Soboleva and Temirova); columns 4 and 5 , the fluxes with the corresponding errors: $\mathrm{F}_{1}$, the fluxes found by E. K. Majorova using the first method, and $F_{2}$, the fluxes found by N. S. Soboleva and A. V. Temirova using the second method. Columns 6 and 7 give the spectral indices $\alpha_{3.94}$ and $\alpha_{0.5}\left(F_{\nu} \sim \nu^{\alpha}\right)$ of the objects found at two frequencies 3.94 and $0.5 \mathrm{GHz}$ (we chose the $0.5 \mathrm{GHz}$ frequency by analogy 
with [19]). Columns 8-10 contain the comments.

We used the source fluxes determined using the two methods to construct the spectra. To this end, we also used all the known catalogs available via the CATS [20, 21], Vizier [22], and NED [23] databases, which intersect with the band of our sky survey. We approximated the spectra by the curves used to determine the spectral indices of the objects at $3.94\left(\alpha_{3.94}\right)$ and $0.5 \mathrm{GHz}\left(\alpha_{0.5}\right)$.

When constructing the spectra we also used, in addition to the available data from known catalogs, the flux estimates obtained by O. P. Zhelenkova based on the maps of the VLSS (74 MHz) [24] and GB6 (4850 MHz) [25] surveys. These estimates were obtained for the objects of our list missing from the VLSS and GB6 catalogs, because they had fluxes below the $5 \sigma$ level (here $\sigma$ is the r.m.s. error of noise for the given map). sources exceed $5 \sigma$.

Table. A fragment of the RCR catalog at $7.6 \mathrm{~cm}$ in the right-ascension interval $7^{h} \leq$ R.A. $<17^{h}(1980-1999)$

\begin{tabular}{c|c|c|c|c|c|c|l|l|l}
\hline $\begin{array}{c}\text { R.A.2000 } \begin{array}{c}D e c_{2000} \\
\text { NVSS }\end{array} \\
\text { (1) }\end{array}$ & $\begin{array}{c}\Delta \mathrm{RA}_{1} \pm \sigma \\
\text { s.ss } \quad \text { s.ss }\end{array}$ & $\begin{array}{c}\Delta \mathrm{RA}_{2} \pm \sigma \\
\text { s.ss }\end{array}$ s.ss & $\begin{array}{c}\mathrm{F}_{1} \pm \sigma \\
\mathrm{mJy}\end{array}$ & $\begin{array}{c}\mathrm{F}_{2} \pm \sigma \\
\mathrm{mJy}\end{array}$ & $\alpha_{3.94}$ & $\alpha_{0.5}$ & pr1 & pr2 & pr3 \\
\hline $070209.13+044011.3$ & $1.03 \pm 0.51$ & $-1.97 \pm 0.84$ & $247 \pm 25$ & $174 \pm 10$ & -0.98 & -0.72 & & & \\
$070210.69+044837.1$ & $2.59 \pm 0.50$ & & $19.4 \pm 1.4$ & & -0.68 & & & & $\#$ \\
$070309.94+045510.7$ & $1.00 \pm 0.42$ & $0.70 \pm 0.88$ & $13.1 \pm 3.0$ & $14.2 \pm 2.0$ & -0.61 & & & $*$ & $\#$ \\
$070424.60+050418.7$ & $-0.31 \pm 0.36$ & $0.66 \pm 0.27$ & $26.6 \pm 4.0$ & $19.3 \pm 3.3$ & -0.30 & & & $*$ & \\
$070451.88+050358.9$ & $0.00 \pm 0.73$ & $0.06 \pm 0.94$ & $18.4 \pm 3.5$ & $24.3 \pm 2.4$ & 0.01 & & $\mathrm{~d} \mathrm{G}$ & $*$ & $\#$ \\
$070612.43+045546.8$ & $0.33 \pm 0.54$ & $0.14 \pm 0.41$ & $8.2 \pm 1.2$ & $9.3 \pm 2.1$ & -0.34 & & & $*$ & $\#$ \\
$070745.79+045525.9$ & & & & & & & $\mathrm{~b}$ & $*$ & $\#$ \\
$070747.30+045414.0$ & $0.92 \pm 0.43$ & $0.51 \pm 0.10$ & $21.7 \pm 2.1$ & $22.9 \pm 2.7$ & -0.19 & & $\mathrm{~b} \mathrm{G}$ & & $\#$ \\
$071130.04+045140.0$ & $0.04 \pm 0.30$ & $0.42 \pm 0.32$ & $33.4 \pm 3.0$ & $39.0 \pm 3.9$ & -0.79 & & $\mathrm{~b} \mathrm{G}$ & & $\#$ \\
$071130.37+050037.0$ & $0.37 \pm 0.30$ & $0.75 \pm 0.32$ & & & & & $\mathrm{~b}$ & & $\#$ \\
\hline
\end{tabular}




\begin{tabular}{c|c|c|c|c|c|c|c|c|c}
\hline$(1)$ & $(2)$ & $(3)$ & $(4)$ & $(5)$ & $(6)$ & $(7)$ & $(8)$ & $(9)$ & $(10)$ \\
\hline $071141.16+045416.2$ & $-0.29 \pm 0.27$ & $0.08 \pm 0.80$ & $8.3 \pm 1.4$ & $10.0 \pm 1.0$ & -0.20 & & & $*$ & $\#$ \\
$071350.65+050210.2$ & $0.53 \pm 0.25$ & $0.01 \pm 0.13$ & $29.8 \pm 4.8$ & $23.2 \pm 4.2$ & -0.69 & -0.69 & $\mathrm{~V}$ & & \\
$071414.01+045526.9$ & $0.54 \pm 0.10$ & & $11.0 \pm 6.0$ & & -0.57 & & $\mathrm{G}$ & $*$ & $\#$ \\
$071616.25+050016.3$ & $-0.65 \pm 0.51$ & $2.19 \pm 0.75$ & $10.0 \pm 1.5$ & $9.7 \pm 1.8$ & -0.19 & & $\mathrm{G}$ & & $\#$ \\
$071833.98+045632.9$ & $-0.06 \pm 0.48$ & $0.13 \pm 0.35$ & $35.7 \pm 5.0$ & $20.5 \pm 4.5$ & -0.75 & -0.75 & $\mathrm{~b}$ & & \\
$071834.62+045248.0$ & $0.58 \pm 0.50$ & $0.77 \pm 0.35$ & & & & & $\mathrm{~b} \mathrm{G}$ & & \\
$071900.99+044705.2$ & $-0.06 \pm 1.40$ & $1.56 \pm 0.71$ & $51.4 \pm 6.7$ & $38.4 \pm 5.3$ & -1.06 & -1.06 & & & \\
$071910.80+045746.6$ & $0.67 \pm 0.32$ & $1.17 \pm 0.45$ & $9.5 \pm 1.5$ & $14.6 \pm 4.2$ & -0.52 & & & $*$ & $\#$ \\
$072043.38+045026.1$ & $2.06 \pm 1.30$ & $0.26 \pm 0.63$ & $28.2 \pm 4.2$ & $21.3 \pm 4.7$ & -0.27 & & $\mathrm{G}$ & & $\#$ \\
$072219.15+045455.4$ & $-0.11 \pm 0.31$ & $-0.16 \pm 0.18$ & $23.9 \pm 2.2$ & $22.2 \pm 2.1$ & -0.29 & & $\mathrm{G}$ & & $\#$ \\
$072318.92+045535.0$ & $-0.13 \pm 0.37$ & $-0.03 \pm 0.59$ & $11.8 \pm 1.3$ & $16.3 \pm 1.0$ & -0.91 & -0.91 & $\mathrm{G}, \mathrm{V}$ & $*$ & $\#$ \\
$072415.87+044525.1$ & $1.20 \pm 0.83$ & $2.02 \pm 0.61$ & $78.6 \pm 2.8$ & $60.5 \pm 3.1$ & -0.71 & -0.87 & & & \\
$072500.61+045008.9$ & $-0.16 \pm 0.25$ & $0.34 \pm 0.78$ & $20.7 \pm 3.0$ & $16.0 \pm 1.3$ & -0.37 & & & $*$ & $\#$ \\
\hline
\end{tabular}

The \# sign in column 10 indicates the objects with flux data available only from two catalogsNVSS and RCR (RC). Part of these objects have flux estimates based on the VLSS and GB6 maps.

The $4850 \mathrm{MHz}$ flux estimates based on GB6 maps made it possible to corroborate the reliability of the extraction of faint objects from our scans. The 74-MHz flux estimates (VLSS maps) confirmed the linear form of the spectra of radio sources in a number of cases. However, we did not use these estimates to find the spectral index at $500 \mathrm{MHz}$ for the sources with no flux data at frequencies below 1.4 GHz. For this reason, we do not give the corresponding spectral indices $\alpha_{0.5}$ in the Table even for objects with linear spectra.

We extracted a total of 550 objects in the right-ascension band $7^{h} \leq$ R.A. $<17^{h}$ including 18 blends and 15 double sources. All these objects are identified with NVSS sources. For 245 sources the fluxes are known only at two frequen- cies $(3.94 \mathrm{GHz}-\mathrm{RATAN}-600$ and $1.4 \mathrm{GHz}-\mathrm{VLA}$ ). Some of them have been corroborated by 4.85 GHz flux estimates based on GB6 maps or 7.7 and $11.111 \mathrm{GHz}$ RATAN-600 data obtained during the same observation sets.

The catalog has a gap in the region $16^{h} 50^{m} 55^{s}<$ R.A. $<16^{h} 51^{m} 30^{s}$, since objects located in this area are blended with the bright extended radio galaxy Hercules A. In addition, the RCR catalog has about one-minute wide gaps at the beginning of each hour due to the calibration of the radiometer.

\section{RELIABILITY OF THE RCR OBJECTS}

\section{IDENTIFICATION}

To assess the reliability of the identification of RCR objects with the objects of the NVSS catalog, we used the data for the entire list of sources in the right-ascension range $7^{h} \leq$ R.A. $<$ 
$17^{h}$.

We computed the NVSS minus RCR rightascension differences $\Delta \mathrm{RA}_{1}, \Delta \mathrm{RA}_{2}$ for the sources and constructed the corresponding histograms for the first and second methods of data reduction and for the averaged $\Delta \mathrm{RA}_{\text {mean }}$. We present these histograms in Fig. 3.

Figures $3 \mathrm{a}-3 \mathrm{c}$ show the histograms of the $\Delta \mathrm{RA}_{1}=\mathrm{R} \cdot \mathrm{A} \cdot N V S S-\mathrm{R} \cdot \mathrm{A}_{1}, \Delta \mathrm{RA}_{2}=$ R.A. ${ }_{N V S S}-\mathrm{R} . \mathrm{A} \cdot 2$, and $\Delta \mathrm{RA}_{\text {mean }}=\mathrm{R} \cdot \mathrm{A} \cdot{ }_{N V S S}-$ R.A.mean right-ascension differences, where, $\mathrm{R} . \mathrm{A} \cdot$ mean $=(\mathrm{R} \cdot \mathrm{A} \cdot 1+\mathrm{R} \cdot \mathrm{A} \cdot 2) / 2$.

In addition, we estimated the mean $\overline{\Delta \mathrm{RA}}$ averaged over the entire sample of sources and the two methods of data reduction. The mean $\overline{\Delta \mathrm{RA}}$ values averaged over the entire sample are equal to:

$\overline{\Delta \mathrm{RA}_{1}}=(0.63 \pm 1.11), \mathrm{s}$,

$\overline{\Delta \mathrm{RA}_{2}}=(0.29 \pm 1.08) \mathrm{s}$, and

$\overline{\Delta \mathrm{RA}_{\text {mean }}}=(0.52 \pm 1.05) \mathrm{s}$.

For the sources with well-studied spectra and known fluxes at several frequencies we determined the expected $3.94-\mathrm{GHz}$ fluxes $\mathrm{F}_{\mathrm{sp}}$ and computed the ratios $\mathrm{F}_{\mathrm{sp}} / \mathrm{F}_{1}, \mathrm{~F}_{\mathrm{sp}} / \mathrm{F}_{2}$ and $\mathrm{F}_{\mathrm{sp}} / \mathrm{F}_{\text {mean }}$, where $\mathrm{F}_{1}, \mathrm{~F}_{2}$ и $\mathrm{F}_{\text {mean }}$ are the fluxes of the RCR sources found using the first and second methods and the average of the two estimates. Figure 4 represents the corresponding distributions: 4a the histogram of the $\mathrm{F}_{\mathrm{sp}} / \mathrm{F}_{1}$ ratios; $4 \mathrm{~b}$ the histogram of the $\mathrm{F}_{\mathrm{sp}} / \mathrm{F}_{2}$ ratio, and 4c the histogram of the $\mathrm{F}_{\mathrm{sp}} / \mathrm{F}_{\text {mean }}$ ratio, where $\mathrm{F}_{\text {mean }}=\left(\mathrm{F}_{1}+\mathrm{F}_{2}\right) / 2$.

The median values of the histograms in Fig. 4
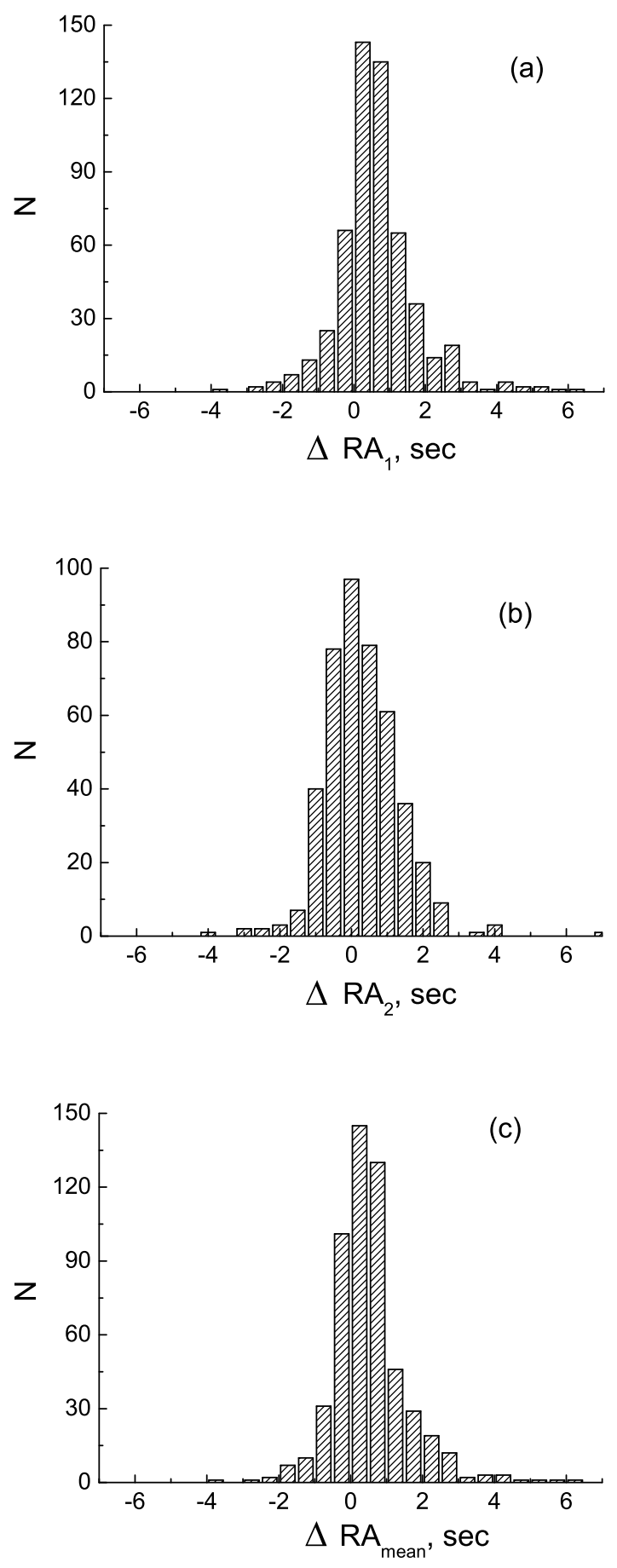

Figure 3. NVSS minus RCR right-ascension differences for RCR sources: $\Delta \mathrm{RA}=\mathrm{R} \cdot \mathrm{A} \cdot{ }_{N V S S}-\mathrm{R} . \mathrm{A} \cdot 1(3 \mathrm{a})$;

$$
\Delta \mathrm{RA}=\mathrm{R} . \mathrm{A} \cdot N V S S-\mathrm{R} . \mathrm{A} \cdot 2(3 \mathrm{~b}) ;
$$

$\Delta \mathrm{RA}=\mathrm{R} \cdot \mathrm{A} \cdot{ }_{N V S}-\mathrm{R} \cdot \mathrm{A} \cdot$ mean $(3 \mathrm{c})$, where

$\mathrm{R} . \mathrm{A} \cdot$ mean $=(\mathrm{R} \cdot \mathrm{A} \cdot 1+\mathrm{R} \cdot \mathrm{A} \cdot 2) / 2$. 

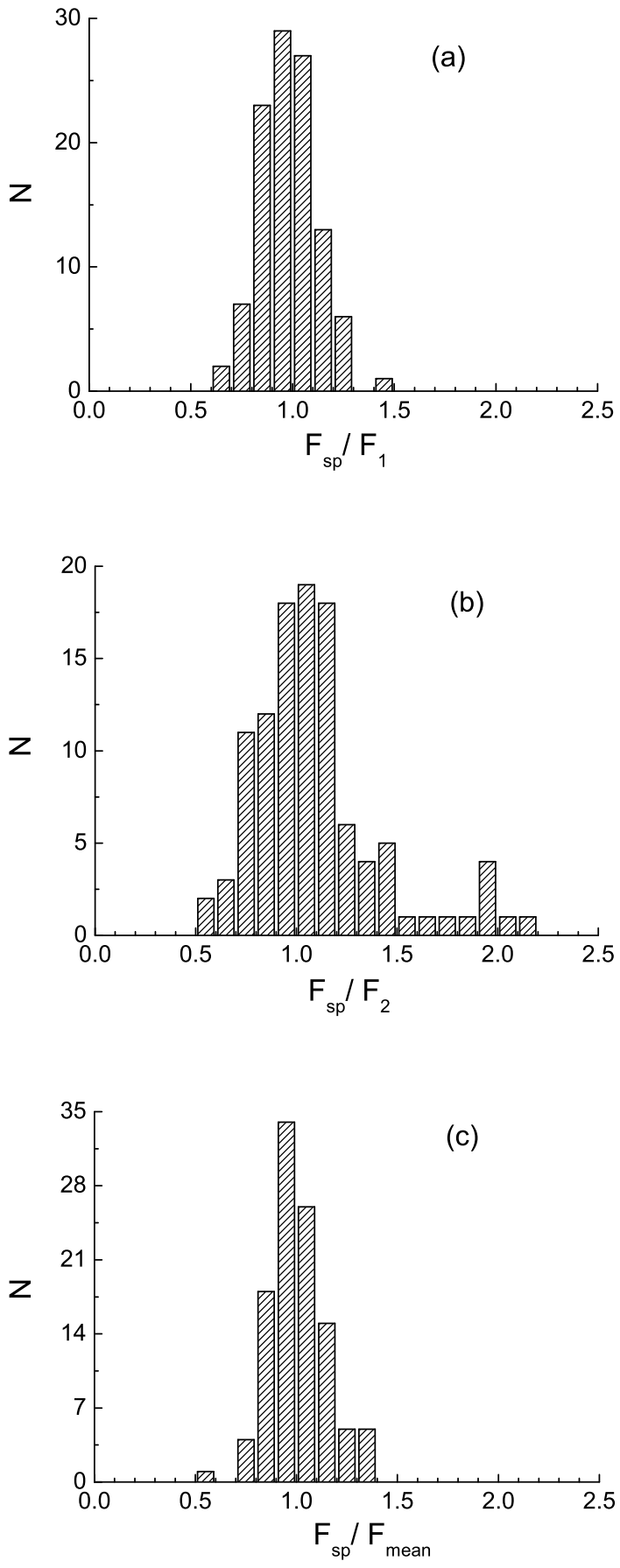

Figure 4. Histograms of the ratios of the expected $3.94 \mathrm{GHz}$ fluxes $F_{s p}$ inferred from the spectra of well studied sources to the fluxes of the same sources determined in this paper using different methods. In fig. 4a we present $\mathrm{F}_{\mathrm{sp}} / \mathrm{F}_{1}$, in $4 \mathrm{~b}: \mathrm{F}_{\mathrm{sp}} / \mathrm{F}_{2}$, and in $4 \mathrm{c}$ :

$$
\mathrm{F}_{\mathrm{sp}} / \mathrm{F}_{\text {mean }} \text {, where } \mathrm{F}_{\text {mean }}=\left(\mathrm{F}_{1}+\mathrm{F}_{2}\right) / 2 \text {. }
$$

are equal to $0.99(4 \mathrm{a}), 1.03(4 \mathrm{~b})$, and $0.99(4 \mathrm{c})$. The mean $\overline{\mathrm{F}_{\mathrm{sp}} / \mathrm{F}}$ ratios averaged over the entire sample are equal to $0.98 \pm 0.01,1.09 \pm 0.03$, and $1.00 \pm 0.01$ for the fluxes found using the first and second methods and the averages of the two estimates, respectively.

Hence the the expected 7.6-cm fluxes based on the spectral data for well-studied radio sources agree best with the halfsum of the fluxes found using two independent methods. The first and second methods yielded somewhat over- and underestimated fluxes, respectively. Recall that the background level is computed with a $80-$ and 20s "smoothing window" in the former and latter cases, respectively.

Thus the use of two different independent methods for extracting the sources and finding their parameters proved to be efficient and justified.

We found the following method to be an efficient tool for testing the reliability of the sources extraction from the scans. All scans are subdivided into two groups with equal number of scans. The scans of each group are then averaged and these averaged scans are used to check whether a particular source is present in the records. The difference between the two scans is then computed and this procedure removes, with a certain accuracy, all the faint objects that are invisible because of the limited sensitivity (the saturation effect) and the difference scan shows only the noise record of the receiver and atmospheric noise. 

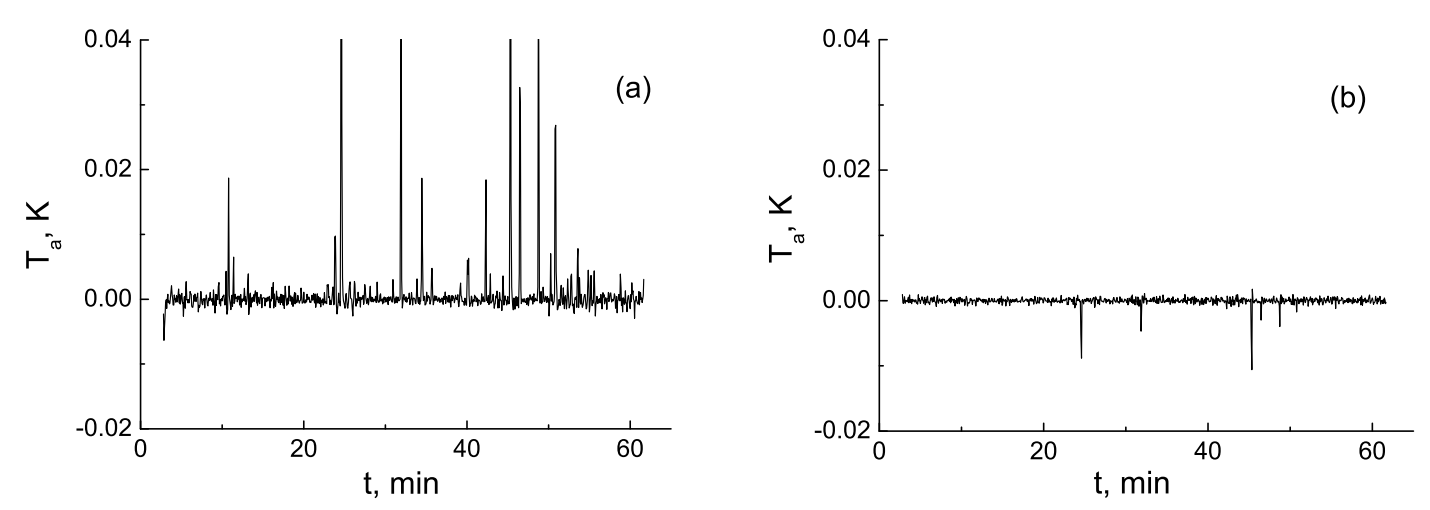

Figure 5. The sum (5a) and difference (5b) of the averaged scans obtained from two groups of 11-h records taken in 1994.

In fig. 5 we present the sum (5a) and difference $(5 \mathrm{~b})$ of the averaged scans inferred from two groups of 11-h records made in 1994. Weak (about 8\%) "negative sources" indicate the measurements accuracy of the objects fluxes. We used this technic in the second method of data reduction.

\section{SPECTRAL INDICES OF THE RCR CATALOG RADIO SOURCES}

In this section we analyze the spectral indices of the radio sources at $3.94 \mathrm{GHz}\left(\alpha_{3.94}\right)$. To this end, we subdivide all RCR sources into three groups.

The first group consists of the sources with known fluxes from other catalogs.

The second group consists of bright sources known from other catalogs and with well-studied spectra with fluxes known at several frequencies. We used the objects of this group to check the reliability of the right ascensions and fluxes find- ings for objects of the RCR catalog (see the previous section). The sources of this group are also included into the first group.

The third group consists of the sources with the data available only from two catalogs-NVSS and $\mathrm{RCR}$ - at the 1.4 and $3.94 \mathrm{GHz}$ frequencies. For some of these sources flux estimates were obtained based on the GB6 (4.85 GHz) and VLSS (74 MHz) maps.

Let us first consider the second group of sources with well-studied spectra and see how their spectra change if we supplement the available data with flux estimates based on the GB6 and VLSS maps.

Figure 6a presents the histogram of the spectral indices of the objects of this group at $3.94 \mathrm{GHz}\left(\alpha_{3.94}\right)$ computed with the available data supplemented by the $4.85-\mathrm{GHz}$ flux estimates (GB6 maps). We found the flux estimates to agree sufficiently well with the available data including our observations at $3.94 \mathrm{GHz}$. If taken into account, the flux estimates based on 

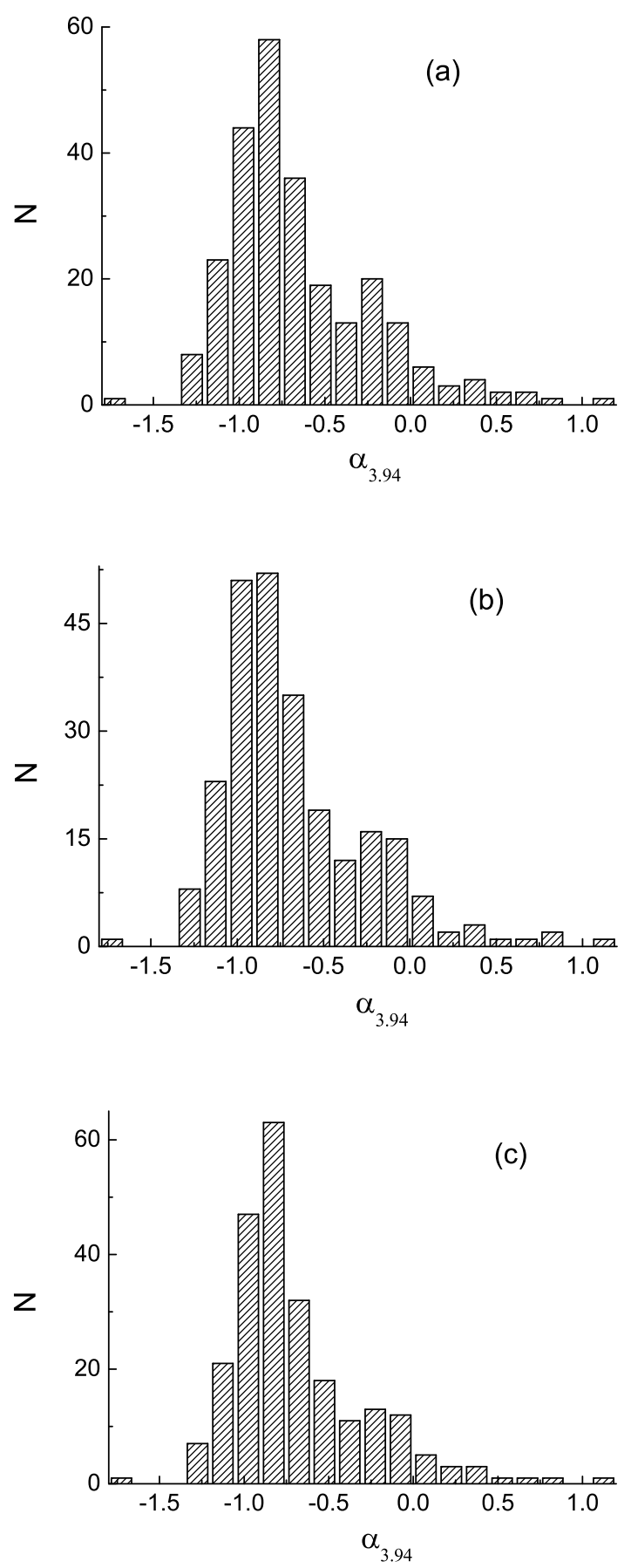

Figure 6. Histograms of spectral indices at $3.94 \mathrm{GHz}$ for the sources of the second group with well-studied spectra: computed taking into account the estimates based on GB6 survey maps (6a), computed taking into account the estimates based on VLSS maps (6b), and computed taking into account the estimates based on GB6 maps and selected estimates based on VLSS maps (6c).
GB6 maps change the spectral indices at this frequency only slightly, on the average by 0.05 . This means that the flux estimates based on GB6 survey maps can also be used to construct the spectra of the sources for that the data are available only at two frequencies (i.e., the sources of the third group). The supplementary data should not change strongly the spectral indices of the sources considered. At the same time, the 4.85$\mathrm{GHz}$ flux estimates provide indirect check of the reliability of our 3.94-GHz data for the sources of the third group.

Figure $6 \mathrm{~b}$ demonstrates the histogram of the spectral indices of the sources of the second group with well-studied spectra after improving the spectra with the flux estimates based on VLSS maps. In a number of cases the use of the 74$\mathrm{MHz}$ flux estimates had virtually no effect on the computed spectral index at $3.94 \mathrm{GHz}$, but in some cases incorporating new data resulted in an insignificant flattening of the spectrum. However, in most of the cases the inclusion of flux estimates made the spectrum steeper at $3.94 \mathrm{GHz}$ and flatter at lower frequencies.

These changes are immediately apparent when we compare Figs. 6a and 6b: the number of sources with the spectral indices equal to -0.8 and -1.0 increased in Fig. $6 \mathrm{~b}$ and so did the number of sources with the spectral index $\alpha_{3.94} \sim-0.15$.

In our subsequent computations of the spectra we used the VLSS map based flux estimates with caution. We used these data primarily in cases 
where they were consistent with the data of other catalogs. In cases where VLSS data were inconsistent with the data of the TXS catalog we preferred the latter or approximated the spectrum by a linear relation.

Figure $6 \mathrm{c}$ represents the distribution of spectral indices $\alpha_{3.94}$ for the same group of sources computed with the flux estimates based on GB6 maps and selected estimates based on VLSS maps taken into account. We list these indices in the Table.

Figure 7a gives us the histogram of the spectral indices $\alpha_{3.94}$ for all objects of the RCR catalog located in the studied right ascension interval $7^{h} \leq$ R.A. $<17^{h}$. Figures $7 \mathrm{~b}$ and $7 \mathrm{c}$ show the histograms of the spectral indices for objects of the first and third groups, respectively.

In addition, we also constructed the histograms of the spectral indices of objects with the antenna temperatures on the averaged scans obeying the conditions $\mathrm{T}_{\mathrm{a}} \geq 5 \sigma$ (Fig. 8a) and $3 \sigma \leq \mathrm{T}_{\mathrm{a}}<5 \sigma$ (Fig. 8b). In the Table the latter sources are marked by asterisks.

It follows from the histograms shown in the figures that most of the objects of the RCR catalog with well-known fluxes at many frequencies have standard power-law spectra with spectral indices in the interval $-1.12<\alpha_{3.94}<-0.5$.

The distributions of spectral indices in Fig. 7a exhibit three well-defined maxima and those shown in Figs. 6, 7b, and 8a-two maxima, that are indicative of the presence of two or more radio sources populations among the objects of our list.
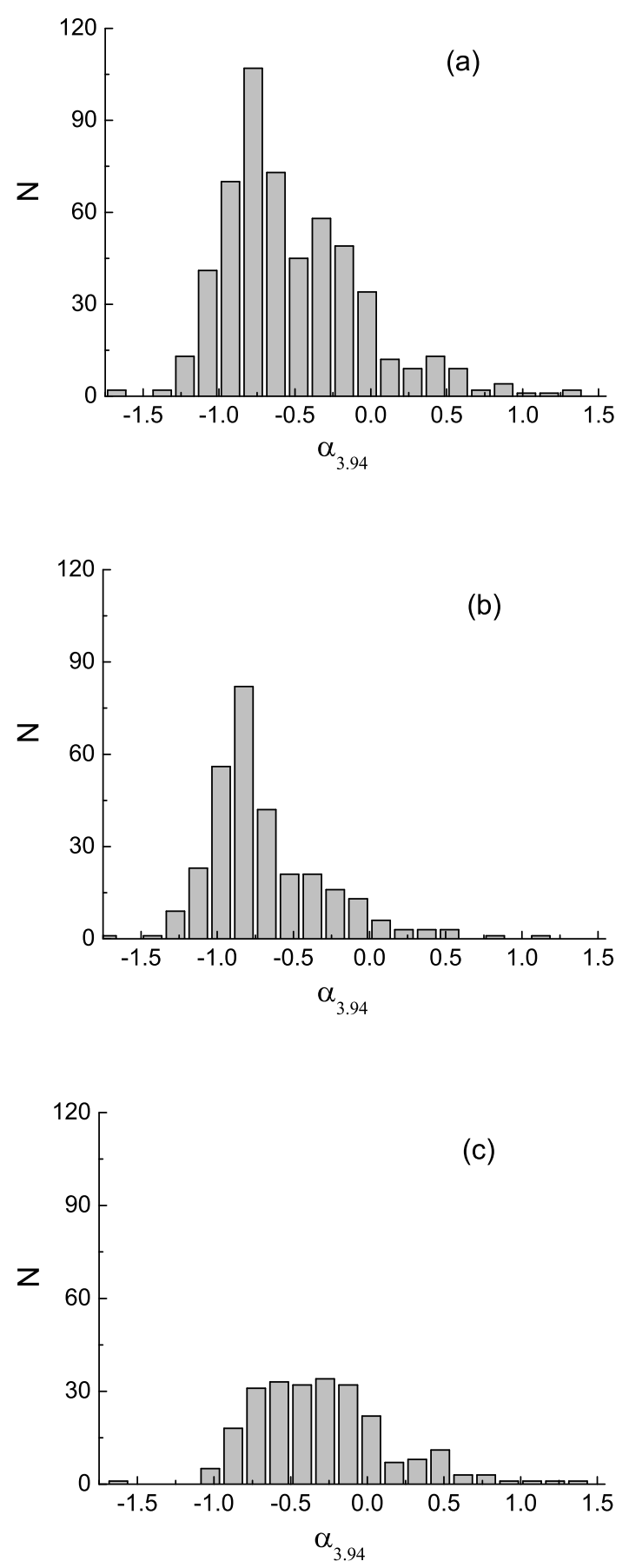

Figure 7. Histograms of spectral indices at 3.94-GHz: (7a) for all sources of the RCR catalog; (7b) for the sources of the first group with flux data available at two or more frequencies, and $(7 \mathrm{c})$ for the sources of the third group with fluxes known only at two frequencies. 

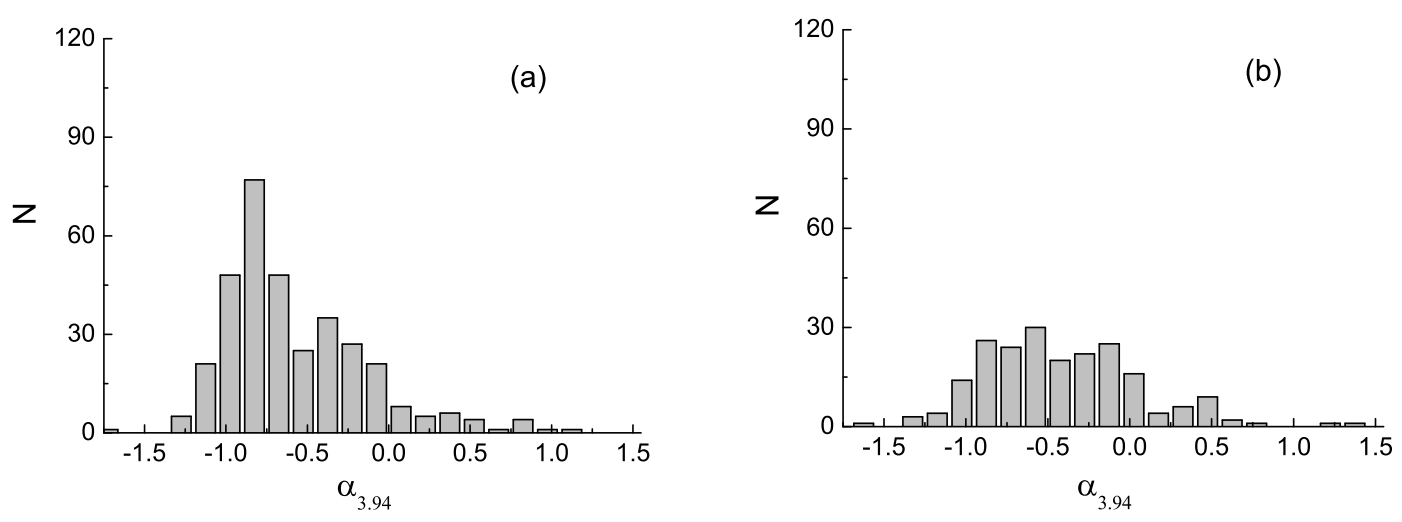

Figure 8. Histograms of spectral indices at $3.94 \mathrm{GHz}$ for sources with antenna temperatures $\mathrm{T}_{\mathrm{a}}>5 \sigma(8 \mathrm{a})$ and $3 \sigma \leq \mathrm{T}_{\mathrm{a}}<5 \sigma(8 \mathrm{~b})$.
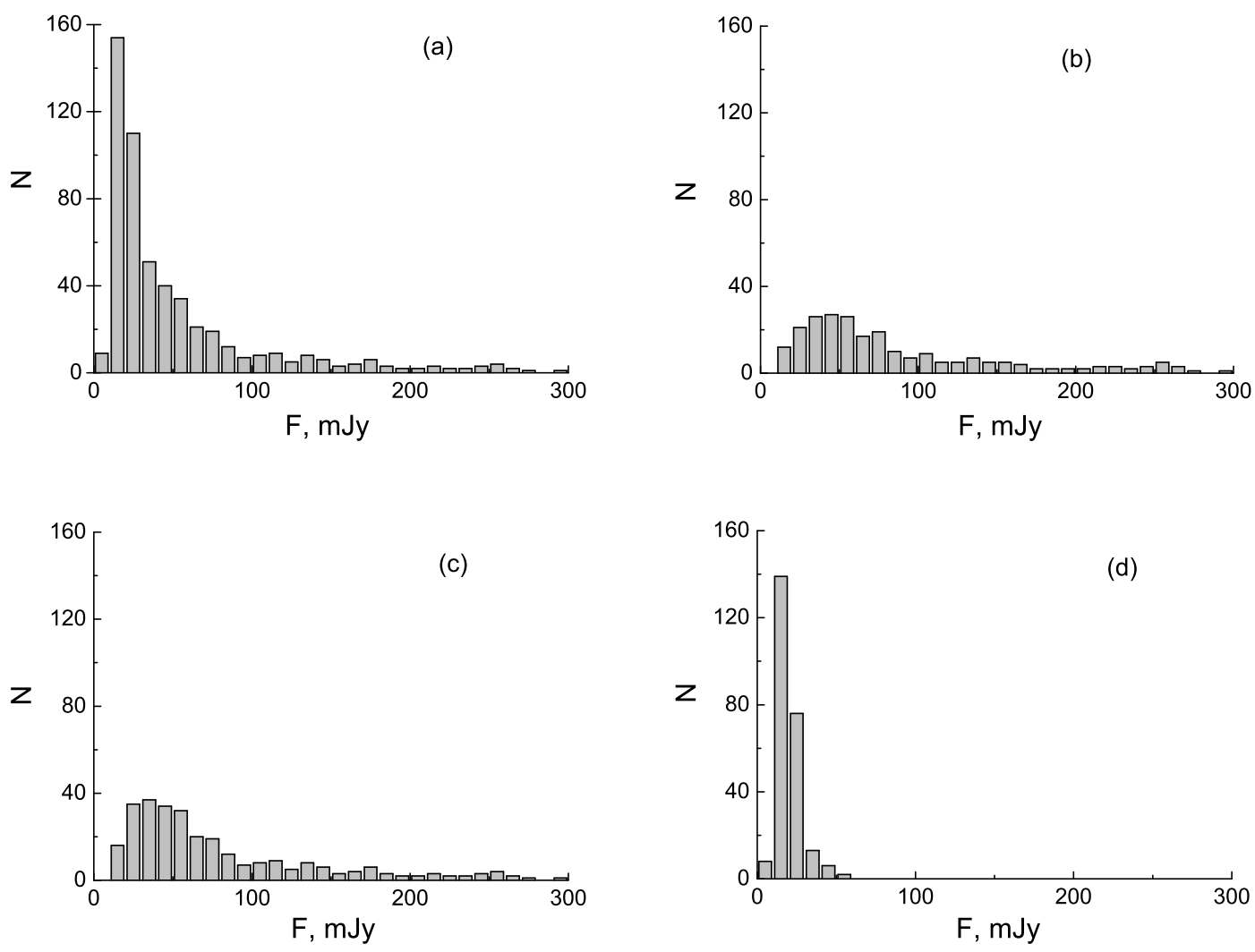

Figure 9. Histograms of 3.94-GHz fluxes for all sources of the RCR catalog (9a), for sources of the second (9b), first (9c), and third (9d) groups.

The maxima in Fig. 7a correspond to the spectral for objects of the third group for that fluxes are indices of $\alpha_{3.94 \max }=-0.8,-0.3$, and +0.45 . known only at two frequencies. The main maxThe second maximum shows up less clearly imum is at $\alpha_{3.94 \max } \sim-0.45$ and the less con- 

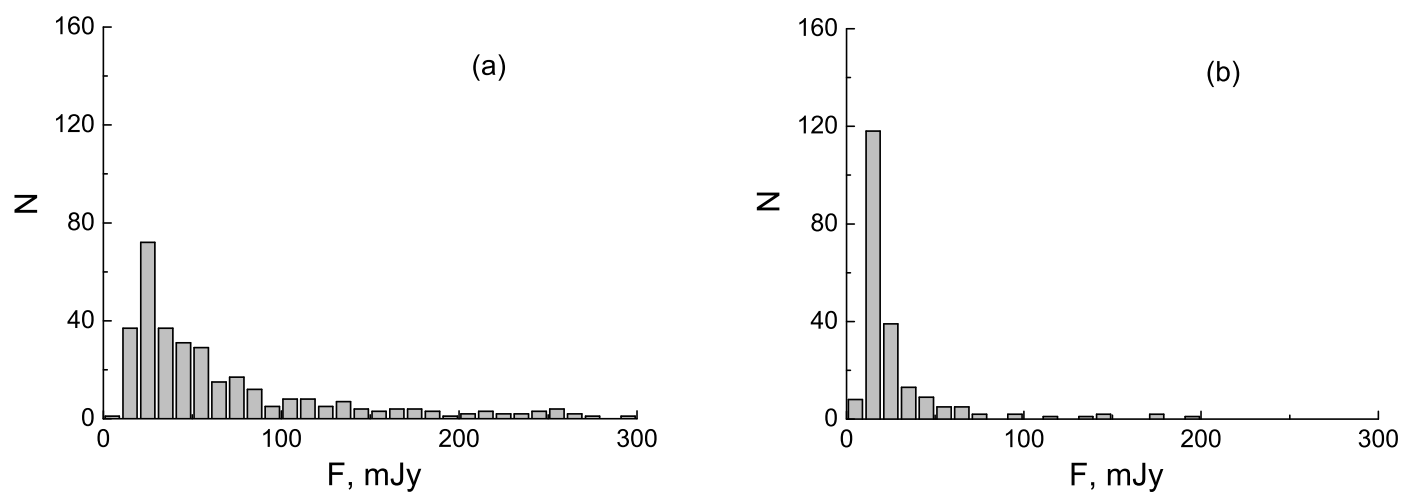

Figure 10. Histograms of the 3.94-GHz fluxes for sources with antenna temperatures $\mathrm{T}_{\mathrm{a}}>5 \sigma(10 \mathrm{a})$ and

$$
3 \sigma \leq \mathrm{T}_{\mathrm{a}}<5 \sigma(10 \mathrm{~b})
$$

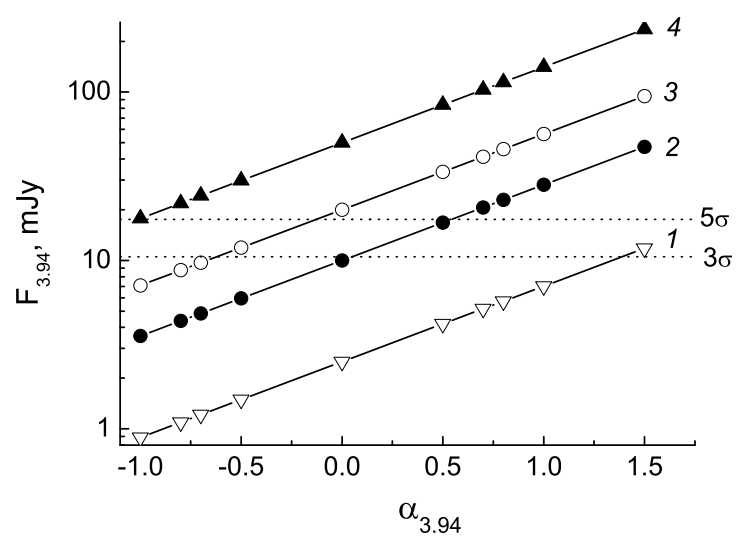

Figure 11. Expected 7.6-cm fluxes for different 1.4-GHz fluxes (NVSS objects) as a function of spectral index. The dotted horizontal lines indicate the threshold of object detection at the $3 \sigma$ and $5 \sigma$ levels for the sensitivity achieved at RATAN-600 radio telescope. The straight lines 1,2, 3, and 4 correspond to NVSS objects with fluxes $\mathrm{F}_{1.4}>2.5 \mathrm{mJy}, \mathrm{F}_{1.4}>10 \mathrm{mJy}, \mathrm{F}_{1.4}>20$ $\mathrm{mJy}$, and $\mathrm{F}_{1.4}>50 \mathrm{mJy}$, respectively. The dependences are given for the central section of the survey.

spicuous maximum is at $\alpha_{3.94 \max } \sim+0.50$.

Objects with antenna temperatures $\mathrm{T}_{\mathrm{a}} \geq 5 \sigma$ (Fig. 8a) exhibit a more conspicuous second maximum in the distribution of spectral indices than

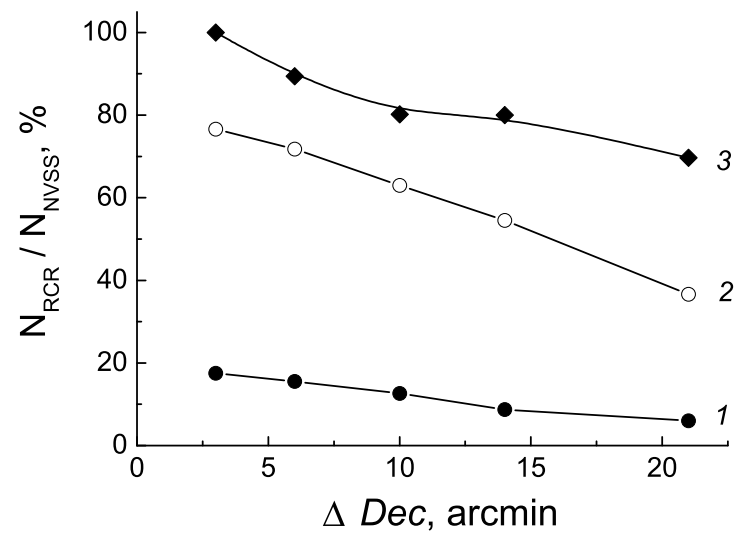

Figure 12. Ratio of the number of RCR objects in the given declination band $D e c_{0} \pm \Delta D e c$ to the number of NVSS sources in the same band as a function of the band width $\Delta D e c$ for several intervals of NVSS object fluxes: $\mathrm{F}_{1.4}<20 \mathrm{mJy}$ (curve 1 ), $20 \mathrm{mJy} \leq \mathrm{F}_{1.4}<100 \mathrm{mJy}$ (curve 2), and $\mathrm{F}_{1.4}>100 \mathrm{mJy}$ (curve 3).

the objects of the first group. Their distribution is more like that of the objects of the second group-i.e., objects with well-studied spectra.

There is also a considerable similarity between the distributions of the spectral indices of objects with antenna temperatures $3 \sigma \leq \mathrm{T}_{\mathrm{a}}<5 \sigma$ and 

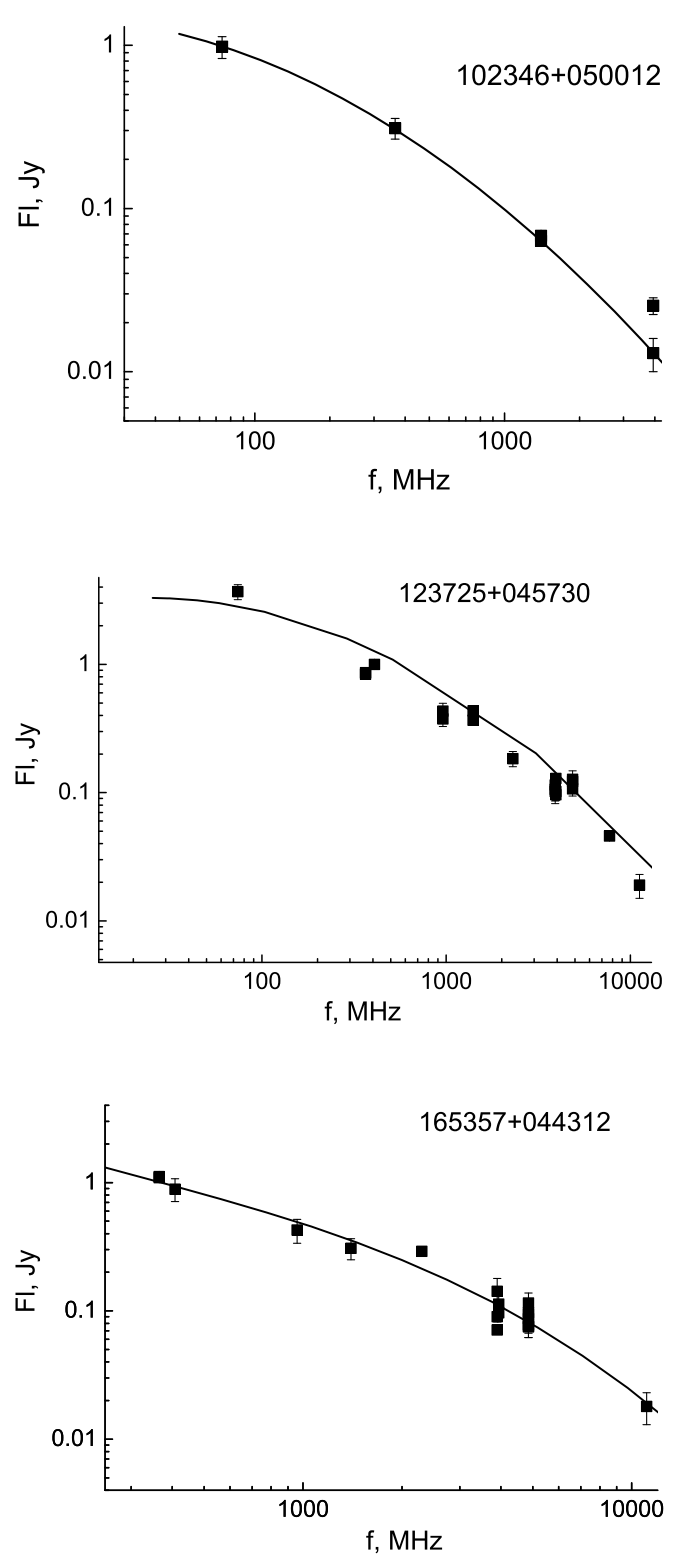

Figure 13. Examples of spectra with the steepness increasing at high frequencies.

objects of the third group, for that the spectral data are known only at two frequencies. Such a similarity is quite natural. The lower the sensitivity threshold $(3 \sigma)$ is, the fainter sources we extract from the records. However, in this case we also face a higher risk of extracting artifacts.

The sample of sources that we extracted at the $3 \sigma-5 \sigma$ level intersects only partially with the sample of objects of the third group. It contains more objects with steep spectra $\left(\alpha_{3.94}<-0.75\right)$ than the sample of objects of the third group, whereas the spectral distributions are almost the same in the $\alpha_{3.94} \geq 0$ interval.

Figure 9 presents the histograms of the 3.94$\mathrm{GHz}$ fluxes for the entire list of the RCR catalog (9a), the sources of the second (9b), first (9c), and third (9d) groups.

Figure 10 demonstrates the histograms of the fluxes of objects with the antenna temperatures $\mathrm{T}_{\mathrm{a}} \geq 5 \sigma$ on the averaged scans (10a) and for sources with $\mathrm{T}_{\mathrm{a}}$ that satisfy the condition $3 \sigma \leq$ $\mathrm{T}_{\mathrm{a}}<5 \sigma(10 \mathrm{~b})$.

The fluxes of the first and second groups sources and the sources with the antenna temperatures $\mathrm{T}_{\mathrm{a}} \geq 5 \sigma$ lie in the interval from $10 \mathrm{mJy}$ to several $\mathrm{Jy}$ (the $\mathrm{x}$-axis domains in the figures are limited by $300 \mathrm{mJy})$.

The fluxes of sources with the antenna temperatures $3 \sigma \leq \mathrm{T}_{\mathrm{a}}<5 \sigma$ also exhibit a large scatter, from 10 to $200 \mathrm{mJy}$. The distribution of the fluxes of this sample objects resembles that of the third group objects. The difference is in that all objects of the third group are faint, with the 3.94-GHz fluxes of 60 mJy or less (Fig. 9d). About $95 \%$ of them have fluxes that do not exceed $30 \mathrm{mJy}$, and most of the sources (about $85 \%$ ) have fluxes in the 10-20 mJy interval.

Number of faint objects with flat or inverse spectra is easy to explain by examining Fig. 11, that demonstrate the changes in the expected 

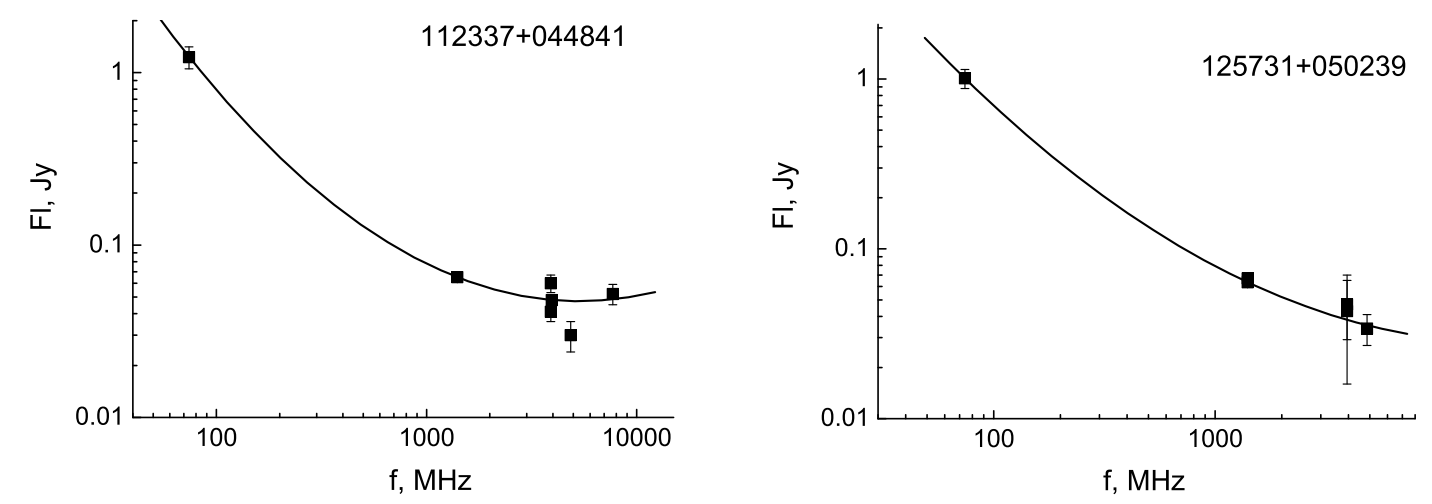

Figure 14. Examples of spectra with the steepness increasing at low frequencies.

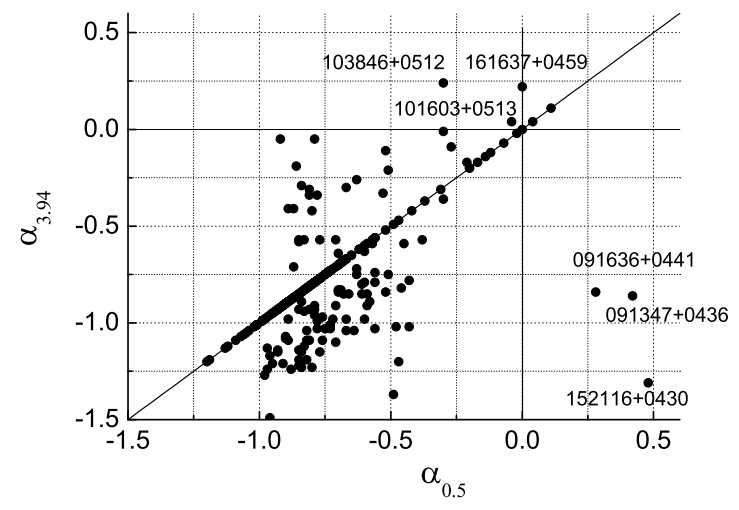

Figure 15. Spectral indices $\alpha_{3.94}$ plotted versus $\alpha_{0.5}$ for the radio sources of the catalog.

7.6-cm fluxes as a function of spectral index for objects with different 1.4-GHz fluxes (NVSS objects).

The horizontal dashed lines in the figure indicate the possibility of objects detection at the $3 \sigma$ and $5 \sigma$ levels for the sensitivity achieved at RATAN-600. The straight lines 1, 2, 3, and 4 correspond to NVSS objects with fluxes $\mathrm{F}_{1.4}>$ $2.5 \mathrm{mJy}, \mathrm{F}_{1.4}>10 \mathrm{mJy}, \mathrm{F}_{1.4}>20 \mathrm{mJy}$, and $\mathrm{F}_{1.4}>50$ mJy, respectively. These dependences are for the central section of the survey.
It follows from Fig. 11 that at the $3 \sigma$ level RATAN-600 could have detected at $7.6 \mathrm{~cm}$ the faintest NVSS objects $\left(2.5 \mathrm{mJy}<\mathrm{F}_{1.4}<10\right.$ mJy) with inverse spectral indices $\alpha>1.5$ (the population of SSA (Synchrotron Self Absorption) and blackbody spectrum objects).

The $3 \sigma$ level in the central band is close to $10 \mathrm{mJy}$, and, starting with a certain integration time, it is virtually independent of further integration due to the saturation effect. We can therefore expect that at this detection level all NVSS objects with fluxes $\mathrm{F}_{1.4}>20 \mathrm{mJy}$ and with spectral indices $\alpha>0$ crossing the central section of our survey can be detected and must have been occurred in the RCR catalog.

We determined the completeness of our catalog from the ratio of the number of objects of the RCR catalog in the given declination interval $D e c_{0} \pm \Delta D e c$ to the number of sources of the NVSS catalog in the same band for several flux intervals of NVSS objects: $\mathrm{F}_{1.4}<20 \mathrm{mJy}$ (curve 1 in Fig. 12), $20 \mathrm{mJy} \leq \mathrm{F}_{1.4} \leq 100 \mathrm{mJy}$ (curve 2 in 

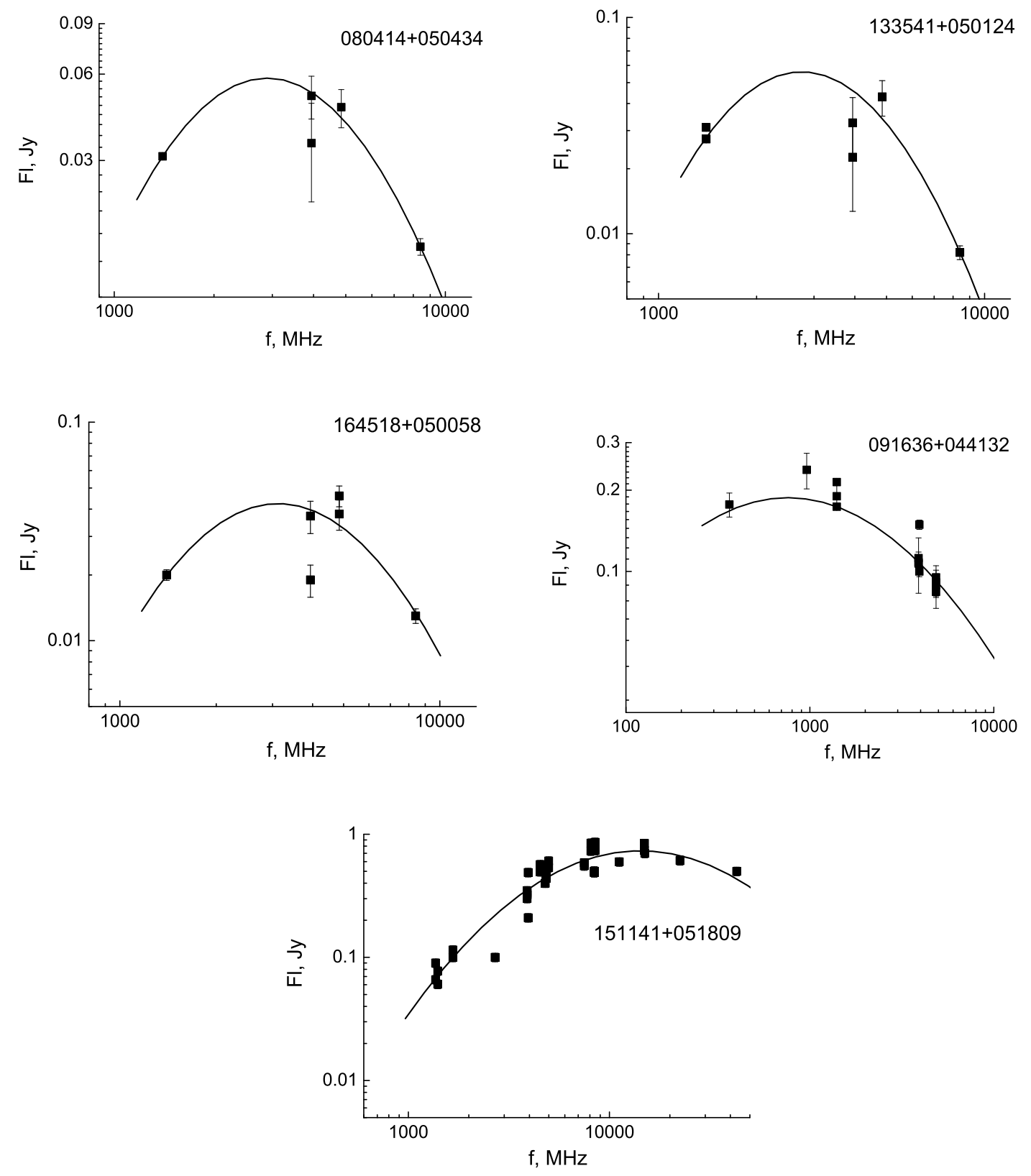

Figure 16. Examples of spectra with a maximum at some frequency. In the Catalog these sources are marked as GPS (column 8).

Fig. 12), and $\mathrm{F}_{1.4}>100$ mJy (curve 3 in Fig. 12). with NVSS objects with fluxes $\mathrm{F}_{1.4} \geq 100 \mathrm{mJy}$,

It follows from Fig. 12 that the complete- $72 \%$ are identified with NVSS objects with fluxes ness of our catalog increases with increasing $20 \mathrm{mJy}<\mathrm{F}_{1.4}<100 \mathrm{mJy}$, and $16 \%$ are identified flux of NVSS objects and with decreasing sur- with NVSS objects with $\mathrm{F}_{1.4} \leq 20 \mathrm{mJy}$. Increasvey band. Thus in the $\Delta D e c= \pm 6^{\prime}$ band a ing the survey band to $\Delta D e c= \pm 20^{\prime}$ reduces total of $90 \%$ RCR sources have been identified the number of sources identified with NVSS ob- 

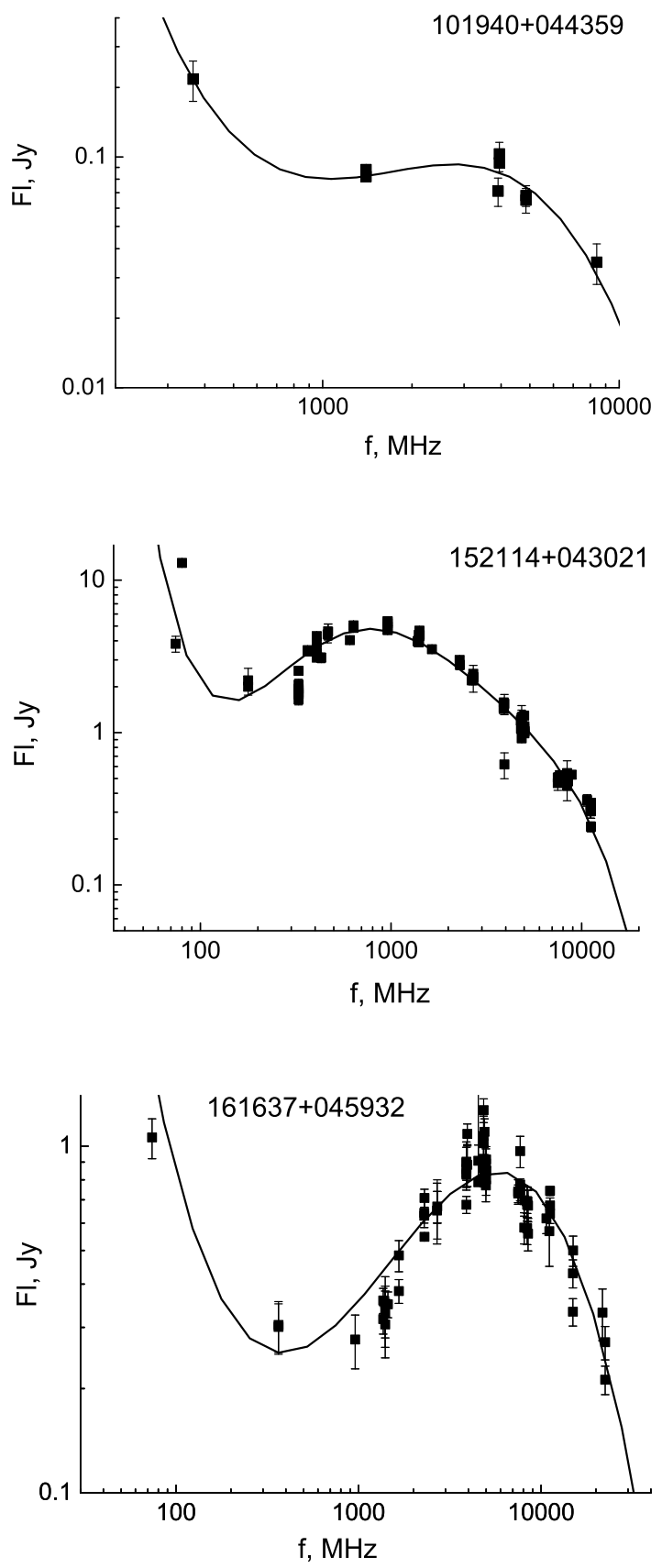

Figure 17. Examples of spectra resulting from a superposition of a power-law spectrum with a self-absorption spectrum at frequencies from 0.5 to $12 \mathrm{GHz}$. In the Catalog objects with the 101940+044359-source-type spectra are marked as "hill" (column 8) (the upper panel).
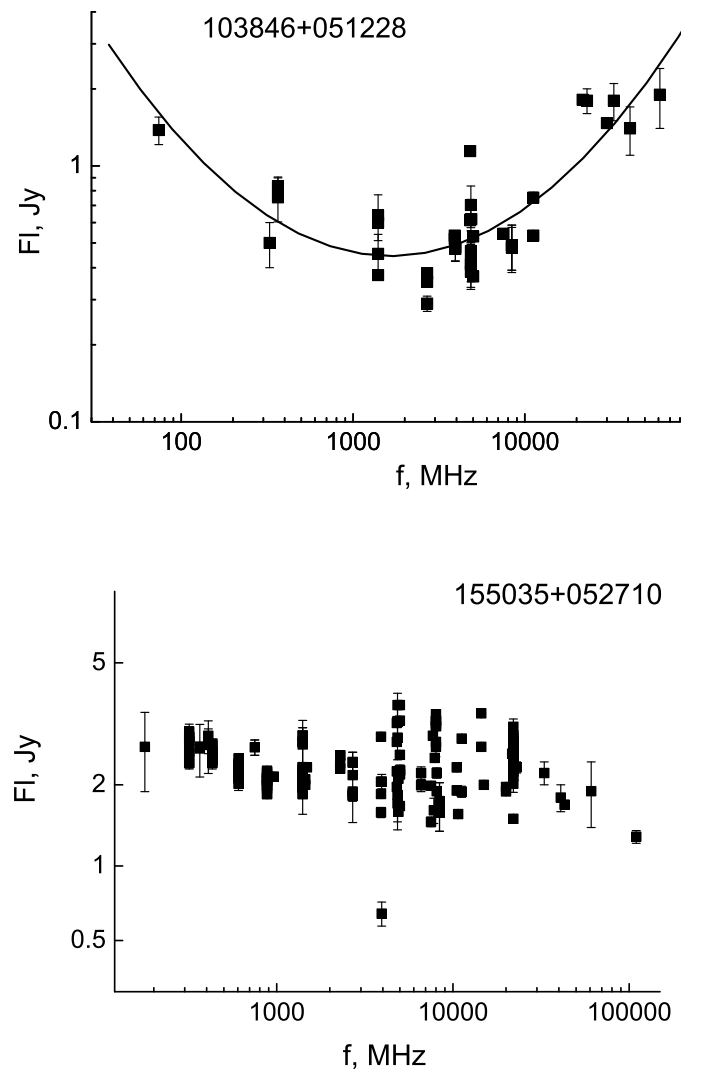

Figure 18. Spectrum with the minimum at a certain frequency (the upper panel) and the spectrum of the variable source (the lower panel).

jects down to $70 \%\left(\mathrm{~F}_{1.4} \geq 100 \mathrm{mJy}\right), 37 \%$ $\left(20 \mathrm{mJy}<\mathrm{F}_{1.4}<100 \mathrm{mJy}\right)$, and $6 \%\left(\mathrm{~F}_{1.4} \leq\right.$ $20 \mathrm{mJy}$ ), respectively.

\section{SPECTRA OF THE RCR CATALOG RADIO SOURCES}

We make it clear from the start that we cannot report the spectra of all radio sources of our list in this paper. We will publish them in a special report and at the http://www.sao.ru/hq/len web page. Here we classify the spectra obtained and discuss the 
most important spectra. Below we present examples of spectra.

Some of the spectra exhibit a sharp maximum in the frequency interval considered. In our Catalog we mark the sources with such spectra as hill, HFP, or GPS (column 8).

Most of the NVSS objects in the RCR catalog with fluxes known at many frequencies have standard power-law spectra with spectral indices in the interval $-1.12<\alpha_{3.94}<-0.5$. These spectra can be fitted quite well by linear relations.

About $20 \%$ of the sources have power-law spectra that become steeper at high frequencies (Fig. 13). Such spectra are usually interpreted as a result of energy loss by energetic relativistic electrons via radiative cooling.

About $10 \%$ of objects have spectra that become flatter at high frequencies (Fig. 14). For most of these objects fluxes are known only at three frequencies: $0.74 \mathrm{GHz}$ (VLSS), $1.4 \mathrm{GHz}$ (NVSS), and $3.94 \mathrm{GHz}$ (RCR); $0.74 \mathrm{GHz}$ fluxes are often just estimates based on the maps of the VLSS survey. The spectra of such sources are believed to be a result of the superposition of a common power-law spectrum from extended components of the radio source with the spectrum from small angular size features (jets emerging from the nucleus) with synchrotron self absorption by relativistic electrons at higher frequencies. In principle, extended components may have different spectra (e.g., steep plus flat), however, they usually have similar spectra and this is unlikely.

We pointed out above that most of the ob- jects have power-law spectra with $\alpha_{3.94} / \alpha_{0.5}=1$. In Fig. 15, where we plot spectral indices $\alpha_{3.94}$ versus $\alpha_{0.5}$, these sources lie along the $\alpha_{3.94} / \alpha_{0.5}$ line. There are a total of about 160 sources with linear spectra; about 70 sources with spectra that become steeper at high frequencies, and about 25 sources with spectra that become steeper at low frequencies. In the domain of negative spectral indices in Fig. 15 the former and latter lie below and above the $\alpha_{3.94} / \alpha_{0.5}$ line, respectively.

The fourth type of spectra are those with a well-defined maximum in the frequency interval from 0.5 to $12 \mathrm{GHz}$. We present examples of such spectra in Figs. 16 and 17. In the literature compact sources with maxima in the spectra are subdivided into three groups: CSS (Compact Steep Spectrum) objects with the maxima at frequencies lower 0.5 GHz; GPS (Gigahertz Peak Spectrum) sources with the maxima at frequencies between 0.5 and $5 \mathrm{GHz}$, and HFP (High Frequency Spectrum) sources with the maxima at frequencies higher $5 \mathrm{GHz}$. Sources with a maximum in the spectrum are believed to be either young objects or blazars [26-28].

The RCR catalog contains 19 objects with the spectra of the fourth type. A minor part of these objects (six sources) have spectra in the form of the superposition of a power-law spectrum at low frequencies onto a self-absorption spectrum at frequencies from 0.5 to $12 \mathrm{GHz}$.

Two out of the six objects with such spectra 
are well-studied radio sources ${ }^{2}$.

Dallacasa et al. [31] identify the GPS radio source $152114+043020$ (or $4 \mathrm{C}+04.51$ ) with a $\mathrm{z}=$ 1.3 galaxy with a compact binary structure on a parsec-size scale. Stangellini et al. [32] classify this source as a quasar. The maximum in its spectrum is located at $1 \mathrm{GHz}$ and its magnitude is $m_{r}=21.2^{m}$.

The $161637+045932$ object from the list of bright HFP sources [33] is a quasar with a redshift $\mathrm{z}=3.22$, angular size $0.76^{\prime \prime}, m_{r}=19.3^{m}$, and spectral maximum located at about $5 \mathrm{GHz}$. Tinti et al. [28] classify this source as a blazar rather than a young object.

For the remaining four objects we found no published evidence indicative of their classification with a certain group. These include two starlike objects: 114631+045818 (with a radio size of $2.27^{\prime \prime}$, a spectral maximum at about $5 \mathrm{GHz}$, and $m_{r}=19.9^{m}$ ) and $101940+044359$ (with a radio size of $3.36^{\prime \prime}$ and $\left.m_{r}=20.1^{m}\right)$ and two galaxies: $125755+045917$ $\left(90^{\prime \prime}, m_{r}=19.1^{m}\right)$ and 091636+044132 (94.2", $\left.m_{r}=16.8^{m}, \mathrm{z}=0.18\right)$. In fig. 17 one can find the spectra of the sources $101940+044359$, $152114+043020$, and $161637+045932$.

No low-frequency fluxes are known so far for other objects with the maxima in the spectra. One of them, $151141+051809$, has a spectral

\footnotetext{
${ }^{2}$ We give the angular sizes of the radio sources according to the FIRST [29] catalog and their r-band magnitudes, according to the SDSS survey [30]. We give the major axis of the ellipse inferred from the Gaussian model of the radio source.
}

maximum at the frequency about $12 \mathrm{GHz}$ with a flux of $1 \mathrm{Jy}$ and is a yet another HFP-type source $[28,33]$. It is a nearby bright $\left(m_{r}=16.3^{m}\right)$ Sy1type galaxy with a redshift of $\mathrm{z}=0.08$.

The other objects of the fourth group are fainter. Their spectral maxima vary from 1.4 to $8 \mathrm{GHz}$. Optically these sources are identified with quasars, starlike objects and with galaxies. Only one of these objects was found in an empty field (EF). For quasars and bright nearby galaxies their spectroscopic redshifts are known. One of these objects is a blazar $154418+045822$ (RX $\mathrm{J} 15442+0458)$ with an angular size of $1.54^{\prime \prime}$ and $r_{m}=18.3^{m}[34]$.

In the Australian Southern-sky catalog at $20 \mathrm{GHz}$ [35] the number of objects of the fourth type with spectral maxima in the $4-8 \mathrm{GHz}$ interval exceeds $20 \%$ of the total number of sources. The detection level of the catalog is $0.5 \mathrm{Jy}$. Massaridi et al. [36] used the results of observations of the Australian telescope at 4.8 and $8.6 \mathrm{GHz}$ and the data from other catalogs at the frequencies of 1.4 and $0.84 \mathrm{GHz}$. The fraction of sources of the fourth type in this catalog [36] is several (about five) times greater than in our catalog. Similarly to the RCR catalog case, this discrepancy cannot be explained by selection.

Four sources of the RCR catalog $(103846+051229, \quad 112417+045144$, $115347+045858 \quad$ and $\quad 133921+050159)$ have spectra that can be approximated fairly well by a parabolas in the 1.4-4.8 Ghz frequency interval. Figure 18 
(the upper panel) presents the spectrum of 103846+051229. It is a triple (according to FIRST data) starlike object $\left(m_{r}=19.1^{m}\right)$ with a faint envelope in its SDSS image.

The $112417+045144$ source consists of a nucleus with a jet and is locate in an empty field. The triple source $115347+045858$ is identified with a nearby galaxy $\left(\mathrm{z}=0.23, m_{r}=17.2^{m}\right)$. The point source $133921+050159$ is identified with a quasar $\left(\mathrm{z}=1.36, m_{r}=19.1^{m}\right)$. In these three sources the nucleus contributes significantly to the overall flux.

Objects with a minimum in the spectrum are called upturn sources [37]. Tucci et al. [38] report the results of the spectral properties analysis for the sources of an almost complete VSA sample (brighter than 10 mJy at $15 \mathrm{GHz}$ ) in the 1.4$33 \mathrm{GHz}$ frequency interval.

The spectra of most of the sources with steep spectra at $1.4-5 \mathrm{GHz}$ proved to become flat with increasing frequency, and the fraction of sources with rising spectra at frequencies above $5 \mathrm{GHz}$ in equal to $19 \%$ of the sample. In our list the former make up for $10 \%$, and the latter (upturn)—only for $0.01 \%$.

This class of sources will possibly be recorded in the future high-resolution observations of the CMB (Cosmic Microwave Background) [38].

Figure 18 (the lower panel) demonstrates an example of the variable source (var) spectrum. Based on the available data, we classified seven sources as variable; all of them have flat spectra. The $073357+045614$ source is possibly variable and it has an inverse spectrum. These are mostly sources dominated by the flux from the nucleus, which are identified with quasars, and some of them $(083148+042938$ [39], 123932+044305 [40], and $142409+043451$ [41]) are classified as blazars. The $101603+051303$ source is in the HPF list [33].

We already pointed out above that about half of all RCR objects have flux data available only at two frequencies 1.4 and $3.94 \mathrm{GHz}$ (plus flux estimates based on GB6 and VLSS maps). These are faint objects with fluxes that are mostly below 30 mJy (Fig. 9b). About 30\% of these objects have power-law spectra with spectral indices ranging from -1 to -0.5 ; about $45 \%$ of all objects have flat spectra, and $25 \%$ objects have inverted spectra. The latter, possibly, have their spectral maxima higher $10 \mathrm{GHz}$.

\section{CONCLUSIONS}

We used RATAN-600 observations of the sky band at the declination $D e c \sim 5^{\circ}$ in the rightascension interval $7^{h} \leq$ R.A. $<17^{h}$ in $1987-1999$ combined with reprocessing of the "Cold" survey data (1980-1981) to compile a list (the RCR catalog) of 550 objects identified with objects of the NVSS catalog. This list includes 18 blends and 15 double sources. Data reduction was performed using two independent methods.

We determined the fluxes, right-ascensions, and spectral indices of every object of our list and constructed the histograms of spectral in- 
dices and fluxes for different samples of sources 3 .

We reconstructed the spectra using all the catalogs available from the CATS, Vizier, and NED databases and the flux estimates based on the maps of the VLSS and GB6 surveys. These estimates are useful primarily for the reconstruction of the sources spectra for that fluxes are known only at two frequencies: $3.94 \mathrm{GHz}(\mathrm{RCR})$ and 1.4 GHz (NVSS). Such objects make up for about $50 \%$ of the RCR catalog (245 objects).

These are mostly sources with fluxes not exceeding $30 \mathrm{Jy}$, about $65 \%$ of them have flat or inverse spectra $(\alpha>-0.5)$. The histograms of the fluxes and spectral indices and the average spectral index of this sample of sources are affected by selection, since objects with steep spectra cannot be detected due to the limited sensitivity of the survey.

We analyzed the reliability of the results obtained. We demonstrated that use of two different methods of data reduction yields more accurate results both for the right ascensions and fluxes of the sources. We therefore believe that the technique of independent reduction of observational data has proved to be successful.

Note in conclusion that the study of spectral indices at centimeter-wave frequencies is closely linked to the problem of eliminating selection effects. For such studies, i.e., for the complete analysis of the spectra of objects in deep

\footnotetext{
${ }^{3}$ We did not determine the declinations of the sources but adopted them from the NVSS catalog.
}

decimeter-wave sky surveys (NVSS [5], FIRST [29]), the sensitivity at centimeter-wave frequencies should be one to two orders of magnitude higher than the sensitivity of decimeter-wave surveys, i.e., at the level of or better than several tens of $\mu \mathrm{Jy}$. So far, such a high sensitivity at centimeter-wave frequencies could have been achieved only within small sky areas.

The aim of sky surveys made with RATAN600 radio telescope $[2,17]$ is to obtain more comprehensive information about the spectral indices of decimeter-wave sources. These surveys serve as an intermediate link between deep VLA surveys and low-sensitivity all-sky surveys. The main and very important conclusion of such surveys is that we found no objects within the right ascension interval considered - at least at the 1015 mJy level-that had not been previously included into decimeter-wave catalogs.

Ninety per cent of the RCR sources identified with NVSS objects and having fluxes $\mathrm{F}_{1.4} \geq 100$ mJy lie in the $\Delta D e c= \pm 6^{\prime}$ band of the survey. In the same band $72 \%$ and $16 \%$ of the sources were identified with NVSS objects with fluxes $20 \mathrm{mJy}<\mathrm{F}_{1.4}<100 \mathrm{mJy}$ and $\mathrm{F}_{1.4} \leq 20 \mathrm{mJy}$, respectively. In the broader band $\Delta D e c= \pm 20^{\prime}$ the number of sources identified with NVSS objects decreases down to $70 \%, 37 \%$, and $6 \%$, respectively.

Thus so far for the small population of NVSS objects all the centimeter-wave surveys including "Cold" and RZF provide the data mostly for objects with synchrotron self absorption (BL Lac, 
QSR, AGN). The new epoch in this field is expected to start the operation of ALMA and SKA instruments at millimiter and about 1-cm waves, respectively.

The catalog is available at http://cdsavc.u-strasbg.fr./viz-bin/

cat?J/other/AstBu/65. 42.

\section{ACKNOWLEDGMENTS}

This work was supported in part by the
ussian Foundation for Basic Research (grant

This work was supported in part by the
Russian Foundation for Basic Research (grant nos. 08-02-00486a and 09-07-00320) and the "Scientific schools" program of the Russian Academy of Sciences. We are grateful to A. I. Kopylov for his critical comments concerning the RC catalog.
1. \refitem $\{$ article $\}$

Yu. N. Parijskij, N. N. Bursov, N. M. Lipovka, et al., A\&AS 87, 1 (1991).

2. $\backslash$ refitem $\{\boldsymbol{m i s c}\}$

Yu. N. Parijskij and D. V. Korol'kov, Itogi Nauki $i$ Tekhniki. Ser. Astronomiya (VINITI, Moscow, 1986) 31, 73 (1986).

3. \refitem $\{$ article $\}$

A. B. Berlin, E. V. Bulaenko, V. Ya. Gol'nev, et al., Pis'ma Astron. Zh. 7, 290 (1981).

4. \refitem $\{$ article $\}$

Yu. N. Parijskij and D. V. Korolkov, Sov. Sci. Rev. Astrophys. Space Rhys. 5, 39 (1986).

5. \refitem $\{$ article $\}$

J. J. Condon, W. D. Cotton, E. W. Greisen, et al., AJ 115, 1693 (1998).

6. \refitem $\{$ article $\}$

R. L. White, R. H. Becker, D. J. Helfand, and M. D. Gregg, Astrophys. J. 475, 479 (1997).

7. $\backslash$ refitem $\{\boldsymbol{m i s c}\}$

O. P. Zhelenkova, Candidate's Dissertation in Mathematics and Physics (Special Astrophysical Observatory of the Russian Academy of Sciences, Nizhnij Arkhyz, 2007).

8. $\backslash$ refitem $\{$ article $\}$

N. S. Soboleva, N. N. Bursov, and A. V. Temirova, Astron. Zh. 83, 387 (2006).

9. $\backslash$ refitem $\{$ article $\}$

N. A. Esepkina, N. L. Kaidanivskii, B. V. Kuznetsov, et al., Radiotekhnika i Elektronika 6, 1947 (1961).

10. \refitem $\{$ article $\}$

N. A. Esepkina, N. S. Bakhvalov, B. A. Vasil'ev, et al., Astrofiz. Issledovaniya (Izv. Spec. Astrofiz. Obs.) 11, 182 (1979).

11. \refitem $\{$ article $\}$

N. A. Esepkina, B. A. Vasil'ev, I. A. Vodovatov, and M. G. Vysotskij, Astrofiz. Issledovaniya (Izv. Spec. Astrofiz. Obs.) 11, 197 (1979).

12. \refitem $\{$ article $\}$

E. K. Majorova, Bull. Spec. Astrophys. Obs. 53, 78 (2002).

13. \refitem $\{$ article $\}$ 
E. K. Majorova and S. A. Trushkin, Bull. 24. \refitem $\{$ article $\}$

Spec. Astrophys. Obs. 54, 89 (2002).

14. \refitem $\{$ article $\}$

E. K. Majorova and N. N. Bursov, Astrophysical Bulletin 62, 398 (2007).

15. $\backslash$ refitem $\{\boldsymbol{u r l}\}$

Skyview, http://skyview.gsf c.nasa.gov/cgi- 26 .

-bin/skvbasic.pl.

16. $\backslash$ refitem $\{$ article $\}$

E. K. Majorova, Astrophysical Bulletin 63, 56 (2008).

17. $\backslash$ refitem $\{$ article $\}$

N. N. Bursov, Yu. N. Pariiskii, E. K. Maiorova, et al., Astron. Zh. 84, 1 (2007).

18. $\backslash$ refitem $\{\boldsymbol{m i s c}\}$

N. S. Soboleva, A. V. Temirova, and N. N. Bursov, Report of the St. Petersburg Branch of the Special Astrophysical Observatory of the Russian Academy of Sciences, No. 2 (2008).

19. $\backslash$ refitem $\{\boldsymbol{u} \boldsymbol{r l}\}$

G. Miley and C. De Breuck, astro-ph/0802.2770

20. $\backslash$ refitem $\{\boldsymbol{m i s c}\}$

O. V. Verkhodanov, S. A. Trushkin, H. Andernach, and V. N. Chernenkov, in Astronomical Data Analysis Software and Systems VI, ed. by G. Hunt and H. E. Payne. ASP Conference Series 125, 322 (1997) (http://cats.sao.ru).

21. $\backslash$ refitem $\{$ article $\}$

O. V. Verkhodanov, S. A. Trushkin, H. Andernach, and V. N. Chernenkov, Bull. Spec. Astrophys. Obs. 58, 118 (2005).

22. \refitem $\{$ article $\}$

F. Ochsenbein, P. Bauer, and J. Marcout, A\&AS 143, 23 (2000).

23. $\backslash$ refitem $\{\boldsymbol{u r l}\}$

NED, http://nedwww.ipac.caltech.edu/.
A. S. Cohen, W. M. Lane, W. D. Cotton, et al., AJ 134, 1245 (2007).

25. \refitem $\{$ article $\}$

P. C. Gregory, W. K. Scott, K. Douglas and J. J. Condon, ApJS 103, 427 (1996).

26. $\backslash$ refitem $\{$ article $\}$

C. P. O'Dea, PASP110, 493 (1998).

27. \refitem $\{$ article $\}$

S. Tinti and G. De Zotti, A\&A 445, 889 (2006).

28. \refitem $\{$ article $\}$

S. Tinti, D. Dallacasa, G. De Zotti, et al, A\&A 432, 31 (2005).

29. \refitem $\{$ article $\}$

R. H. Becker, D. J. Helfand, and R. L. White, AJ 475, 479 (1997).

30. $\backslash$ refitem $\{$ article $\}$

J. K. Adelman-McCarthy et al., ApJS 175, 297 (2008).

31. \refitem $\{$ article $\}$

D. Dallacasa, M. Bondi, W. Alef, and F. Mantovani, A\&AS 129, 219 (1998).

32. \refitem $\{$ article $\}$

C. P. Stanghellini et al., A\&A $\mathbf{4 4 3}, 891$ (2005).

33. \refitem $\{$ article $\}$

D. Dallacasa, C. Stanghellini, M. Centoza, and R. Fanti, A\&A 363, 887 (2000).

34. \refitem\{article\}

S. A. Laurent-Muehleisen et al., AJ 525, 127 (1999).

35. $\backslash$ refitem $\{\boldsymbol{u r l}\}$

M. Massaridi, R. D. Ekers, T. Murphy, et al., astro-ph/0709.3485.

36. \refitem $\{$ article $\}$

M. Massaridi, R. D. Ekers, T. Murphy et 
al., MNRAS (in press).

37. \refitem $\{$ article $\}$

W. A. Dent and F. T. Haddock, Nature 205, 487 (1965).

38. $\backslash$ refitem $\{$ article $\}$

M. Tucci, J. A. Rubio-Martin, R. Rebolo et al., MNRAS 386, 1729 (2008).

39. $\backslash$ refitem $\{$ article $\}$
B. Sbarufatti, A. Treves, and R. Falomo, AJ 635, 173 (2005).

40. \refitem $\{$ article $\}$

G. Nandikotkur et al., AJ 657, 706 (2007).

41. \refitem $\{$ article $\}$

P. S. Chen, H. W. Fu, and Y. F. Gao, New Astronomy 11, 27 (2005). 